University of Louisville

ThinkIR: The University of Louisville's Institutional Repository

Electronic Theses and Dissertations

$5-2011$

\title{
Mixed-effects models for modeling cardiac functions and treatment effects.
}

Hyejeong Jang

University of Louisville

Follow this and additional works at: https://ir.library.louisville.edu/etd

\section{Recommended Citation}

Jang, Hyejeong, "Mixed-effects models for modeling cardiac functions and treatment effects." (2011). Electronic Theses and Dissertations. Paper 679.

https://doi.org/10.18297/etd/679

This Master's Thesis is brought to you for free and open access by ThinkIR: The University of Louisville's Institutional Repository. It has been accepted for inclusion in Electronic Theses and Dissertations by an authorized administrator of ThinkIR: The University of Louisville's Institutional Repository. This title appears here courtesy of the author, who has retained all other copyrights. For more information, please contact thinkir@louisville.edu. 


\title{
MIXED-EFFECTS MODELS FOR MODELING CARDIAC FUNCTIONS AND TREATMENT EFFECTS
}

\author{
By \\ Hyejeong Jang \\ B.S., Chonnam National University, KOREA, 1997
}

\begin{abstract}
A Thesis
Submitted to the Graduate Faculty of

Graduate School of Public Health of the University of Louisville

In Partial Fulfillment of the Requirements

for the Degree of

Master of Science

Department of Bioinformatics and Biostatistics

University of Louisville

Louisville, Kentucky
\end{abstract}

May 2011 



\title{
MIXED-EFFECTS MODELSFOR MODELING CARDIAC FUNCTIONS AND TREATMENT EFFECTS
}

\author{
By \\ Hyejeong Jang \\ B.S., Mathematics education, Chonnam National University, KOREA, 1997
}

A Thesis Approved on

$4 / 19 / 2011$

Date

By the following Thesis Committee:

Thesis Chair : Maying Kong, PhD

Susmita Datta, PhD

Daniel J. Conklin, PhD 


\section{ACKNOWLEDGEMENTS}

This thesis would not have been possible without the guidance from my thesis advisor Dr. Maiying Kong. Her insightful comments and invaluable suggestions have significantly improved the contents of this thesis. I would like to express my gratitude to Dr. Susmita Datta and Dr. Daniel J. Conklin for serving on my thesis committee. I particularly thank Dr. Conklin for providing the experimental data for this thesis. I would like to extend my sincere gratitude to all the professors in the Department of Bioinformatics and Biostatistics who had taught me during the past two years. I learned a lot from these courses, and the courses have equipped me to work on my thesis. I also would like to express my love and deepest gratitude to my family, my son Heeweon Kim, and my husband Seongho Kim for their love and support. Above all, I would like to thank God for giving me the ability, patience and strength to complete this thesis. 


\section{ABSTRACT \\ MIXED-EFFECTS MODELS FOR MODELING \\ CARDIAC FUNCTIONS AND TREATMENT EFFECTS}

Hyejeong Jang

April 15, 2011

Mixed-effects model is an efficient tool for analyzing longitudinal data. The random effects in mixed-effects model can be used to capture the correlations among repeated measurements within a subject. The time points are not fixed and all available data can be used in mixed-effects model provided data are missing at random. For this reason, we focus on applying mixed-effects models to the repeated measurements of cardiac function including heart rate, left ventricle developed pressure, and coronary flow in the Glutathione-S-transferase P (GSTP) gene knockout and wild-type mice following ischemia/reperfusion injury performed in the isolated, Langendorff-perfused heart. Cardiac function is measured during three time periods: pre-ischemia, ischemia (no flow), and reperfusion. We developed piecewise nonlinear mixed-effects model to describe the different aspects of the cardiac function during each period. We applied nonlinear mixedeffects models and a changing point model to examine how cardiac function was altered by ischemia/reperfusion-induced injury and for comparison between mouse strains. These findings provide evidence of a new application for the mixed-effects model in physiological and pharmacological studies of the heart. 


\section{TABLE OF CONTENTS}

PAGE

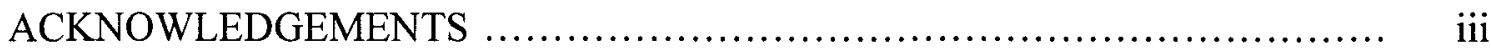

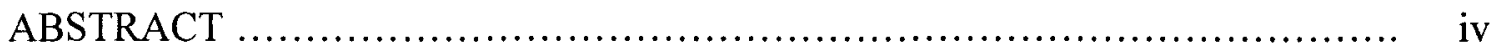

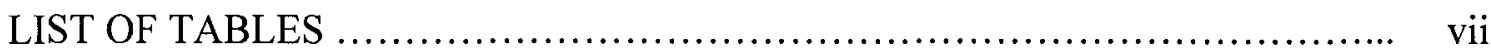

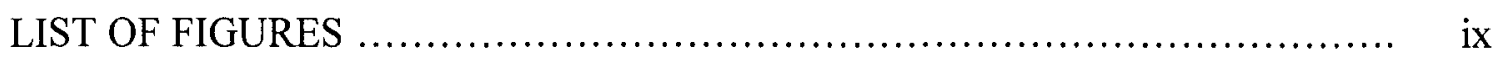

CHAPTER 1. INTRODUCTION ..............................................

1.1 Longitudinal data analysis .........................................

1.2 Mixed-effects models ............................................... 2

CHAPTER 2. MODELS AND METHODS _..................................... 5

2.1 Linear mixed-effects (LME) models ................................... 5

2.2 Nonlinear mixed-effects (NLME) models .............................. 8

2.3 Method of estimation of change point in two phase growth models ........ 11

2.4 Model selection ...................................................... 12

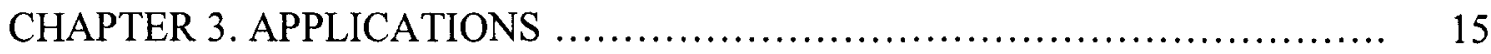

3.1 Experimental data and graphical presentation .......................... 16

3.2 Nonlinear model and statistical analysis for heart rate $\ldots \ldots \ldots \ldots \ldots \ldots \ldots \ldots . \ldots \ldots$

3.3 Nonlinear model and statistical analysis for coronary flow .................. 29

3.4 Nonlinear model and statistical analysis for left ventricle (LV) developed pressure............................................................... 34

CHAPTER 4. DISCUSSIONS AND CONCLUSIONS ........................... 39

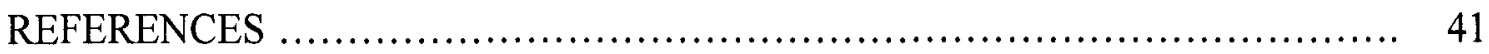


A Linear mixed-effects model for single period ............................. 43

B R code for this thesis .................................................. 49

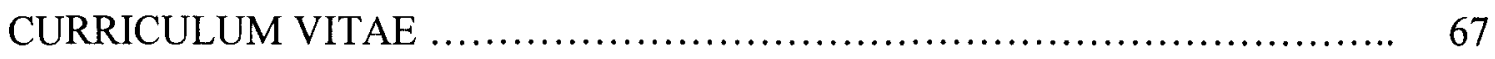




\section{LIST OF TABLES}

TABLE

PAGE

1. Estimates of fixed-effects and variance components for linear mixed-effects model for heart rate during pre-ischemia

2. Estimates of fixed-effects and variance components for nonlinear mixed-effects model (E 3.2) for heart rate during ischemia

3. Estimates of fixed-effects and variance components for nonlinear mixed-effects model (E 3.3) for heart rate during reperfusion

4. Estimates of fixed-effects and variance components for the integrated mixed-effects model (E 3.4) for heart rate

5. Estimates of fixed-effects for the integrated mixed-effects model (E 3.5) for coronary flow

6. Estimates of variance components for the integrated mixed-effects model (E 3.5) for coronary flow

7. Estimates of fixed-effects and variance components for nonlinear mixed effects change point model (E 3.6) for left ventricle (LV) developed pressure during reperfusion

8. Estimates of fixed-effects for linear mixed-effects model (E 3.1) for heart rate during pre-ischemia

9. Estimates of fixed-effects for linear mixed-effects model (E 3.1) for heart rate during ischemia 
10. Estimates of fixed-effects for linear mixed-effects model (E 3.1) for heart rate during reperfusion

11. Estimates of fixed-effects for linear mixed-effects model (E 3.1) for coronary flow during pre-ischemia

12. Estimates of fixed-effects for linear mixed-effects model (E 3.1) for coronary flow during reperfusion

13. Estimates of fixed-effects for linear mixed-effects model (E 3.1) for LV developed pressure during pre-ischemia

14. Estimates of fixed-effects for linear mixed-effects model (E 3.1) for LV developed pressure during reperfusion 


\section{LIST OF FIGURES}

FIGURE $\quad$ PAGE

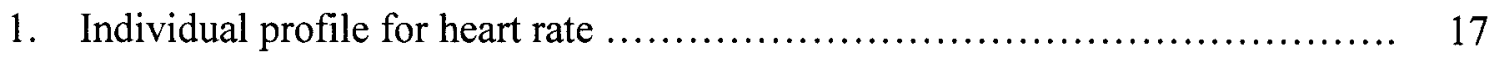

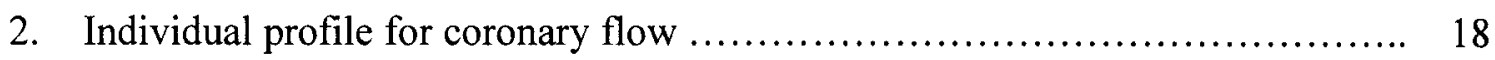

3. Individual profile LV developed pressure .............................. 18

4. Plots of the fitted heart rates at group-level and the predicted heart rates at subject - level, as well as the observed heart rates (circles) versus time during ischemia . 22

5. Plots of the fitted heart rates at group-level and the predicted heart rates at subject - level, as well as the observed heart rates versus time during reperfusion ........ 24

6. Diagnostic plot: Standardized residuals versus subject-level fitted heart rates ....

7. Plots of the fitted heart rates at group-level and the predicted heart rates at subjectlevel, as well as observed heart rates (circles) versus time for the entire experimental data

8. Fitted and observed heart rates versus time 28

9. Diagnostic plot: Standardized residuals versus subject-level fitted coronary flows ...

10. Plots of the fitted coronary flows at group-level and the predicted coronary flows at subject-level, as well as the observed coronary flows (circles) versus time for the entire experimental data

11. Fitted and observed coronary flows versus time

12. Fitted and observed LV developed pressures versus time during reperfusion ..

13. Plots of the fitted LV developed pressures at group-level and the predicted LV 
developed pressures at subject-level, as well as observed LV developed pressures

(circles) versus time during reperfusion

14. Heart rates versus time during pre-ischemia .............................. 43

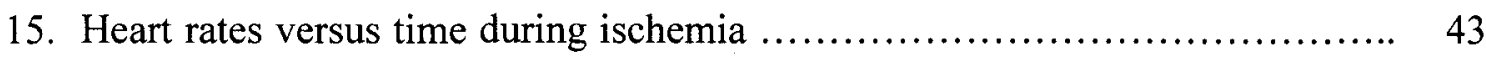

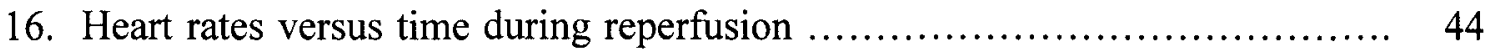

17. Coronary flows versus time during pre-ischemia ......................... 44

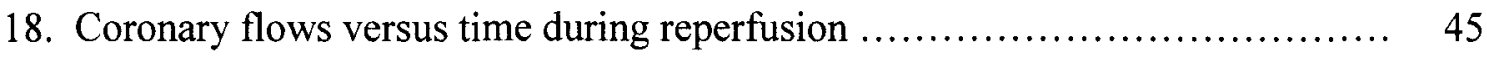

19. Left ventricle (LV) developed pressures versus time during pre-ischemia ..... 45

20. LV developed pressures versus time during ischemia ....................... 46

21. LV developed pressures versus time during reperfusion .................... 46 


\section{CHAPTER 1}

\section{Introduction}

\subsection{Longitudinal data analysis}

Longitudinal studies are defined by having an outcome variable that is repeatedly measured over time. In a longitudinal study, the outcome variable is measured in a subject on several different occasions [1]. Longitudinal studies have been used to study the impact of disease and treatment methods in medicine, to find the cause of the development of children and the clinical progress of patients in psychology, and to study the causes of the behavior change in the behavioral sciences [2].

A longitudinal study has several advantages. First, repeated measurements from the same subject are not completely correlated [3], thus, repeated measures increase statistical power for same or smaller sample size. For this reason, longitudinal studies have more power than cross-sectional studies for a fixed number of subjects [3]. Second, in a longitudinal study, each subject can serve as his or her own control [3]. The exclusion of between-subject variability from measurement error leads to more efficient estimators for parameters related to treatment effects [3]. Third, aging effects (i.e., changes over time within subjects) can be separated from cohort effects (i.e., differences among subjects at at baseline) [3]. As a result, the individual change of a certain outcome 
variable over time can be studied in longitudinal studies [1].

The key feature of longitudinal data is that repeated measures on the same subject are not independent. Therefore, the correlation within a subject must be accounted for using a specific statistical method. Repeated measures analysis of variance (repeated measures ANOVA) and multivariate ANOVA (MANOVA) are two traditional approaches for analyzing longitudinal data [3]. Repeated measures ANOVA is also called a mixed-effects ANOVA, where a single random subject effect is included to account for the correlation among the repeated measurements within the same subject [4]. However, the repeated measures ANOVA requires that all individuals have a complete balanced data set and a fixed time schedule, and treats time as a categorical variable [5]. The advantage of the MANOVA approach versus the ANOVA approach is that the MANOVA assumes a general form for the correlation of repeated measurements over time, while ANOVA assumes a much more restrictive compound-symmetric form [3]. However, MANOVA model has a limitation because it requires complete data [3]. In addition, both MANOVA and ANOVA models provide no information about subjectspecific growth curve [3]. Due to these reasons above, covariance pattern models, generalized estimating equations (GEE) models, and mixed-effects models are generally used for longitudinal data [3].

\subsection{Mixed-effects models}

Mixed-effects models are widely used method for analyzing longitudinal data. Mixed -effects models have been developed and described under various names [3]: random 
effects models [6], variance component models [7], multilevel models [8], hierarchical linear models [9], two-stage models [10], random coefficient models [11], mixed models [12], empirical Bayes models [13], and random regression models [14]. A mixed-effects model has a regression form as linear regression or nonlinear regression, where the coefficients are expressed as summation of fixed effects and random effects. The time points for the measurements of each subject may vary. In a mixed-effects model, random effects are often included to describe the subject-specific effect, and fixed effects are included to describe population-level effect. The correlation between the repeated measurements is captured by the random effects and their distribution assumption. The magnitude of the variance of the random effects captures the between-subject variation. Therefore, mixed-effects models provide a more flexible covariance structure for nonconstant within-subject correlation [15], and have the ability to accommodate missing and imbalanced data which are common in longitudinal data [5].

In many longitudinal analyses, change is assumed to be steady and incremental so that a linear model is appropriate [16]. However, in numerous situations, the change is not uniform but rather faster during some periods and slower in others [16]. In these cases, nonlinear mixed-effects (NLME) models are more appropriate than linear mixed-effects (LME) models to describe these nonlinear changes. A NLME model is usually established based on some mechanistic considerations, and its parameters usually are interpretable. NLME models are often used due to interpretability, parsimony, and validity beyond the observed range of the data [17]. Furthermore, a nonlinear model generally uses fewer parameters than a competitor linear model, providing a more parsimonious description of the data and more reliable predictions for the response 
variables [17].

Based on Cudeck and Harring [16], the best way to select an appropriate NLME model is to graph data from an individual and examine the performance of candidate functions. In lieu of a theoretical rationale that favors a specific form, model choice is made based on the bases of goodness of fit, interpretability of the parameters, and appropriateness of the functional form to the situation in which it is applied.

In this thesis, we applied mixed-effects models and change point model to experimental longitudinal data. The experiments were carried out in Dr. Conklin's laboratory to examine the impact of Glutathione-S-transferase P (GSTP) on the recovery of postischemic cardiac function including heart rate, left ventricle (LV) developed pressure, and coronary flow in the Glutathione-S-transferase P (GSTP) gene knockout (KO) and wild-type (WT) mice following ischemia/reperfusion injury performed in the isolated, Langendorff-perfused heart. Cardiac function was measured during three time periods: pre-ischemia, ischemia (no flow), and reperfusion periods. For this study, we developed piecewise nonlinear function to describe the different aspects of the cardiac function during each period. We applied nonlinear mixed-effects models and changing point model to examine how cardiac function was altered by ischemia/reperfusioninduced injury and for comparison between mouse strains. The Wald test and the Akaike information criterion (AIC) were applied to obtain a parsimonious model for each aspect of cardiac function. 


\section{CHAPTER 2}

\section{Models and Methods}

\subsection{Linear mixed-effects (LME) models}

The linear mixed- effects (LME) model can be written as:

$$
y_{i}=X_{i} \beta+Z_{i} b_{i}+\varepsilon_{i},
$$

where $\left(X_{i}, Z_{i}, y_{i}\right)(i=1, \ldots, N)$ are the observations for $i^{\text {th }}$ subject. Specifically, $X_{i}$ is the $n_{i} \times p$ covariate matrix associated with the fixed effects $\beta$, where $\beta \in R^{p \times 1}, \quad Z_{i}$ is the $n_{i} \times r$ design matrix associated with the random effects $b_{i}$, where $b_{i} \in R^{r \times 1}, y_{i}$ is the $n_{i} \times 1$ vector of response variable, and $\varepsilon_{i}$ is $n_{i} \times 1$ within-subject error vector. The random effects $b_{i}(i=1, \ldots, N)$ are assumed to be independent and identically distributed (i.i.d.) as $N(0, \boldsymbol{D})$, and $\varepsilon_{i}(i=1, \ldots, N)$ are assumed to be i.i.d. as $\varepsilon_{i} \sim N\left(0, \sigma^{2} I_{n_{i}}\right)$, and $b_{i}$ and $\varepsilon_{i}(i=1, \ldots, N)$ are independent. As a result, it can be shown that the observation $y_{i}$ and random effects $b_{i}$ follow the joint multivariate normal distribution:

$$
\left[\begin{array}{c}
y_{i} \\
b_{i}
\end{array}\right] \sim N\left(\left[\begin{array}{c}
X_{i} \beta \\
0
\end{array}\right],\left[\begin{array}{cc}
Z_{i} \boldsymbol{D} Z_{i}^{\prime}+\sigma^{2} I_{n_{i}} & Z_{i} \boldsymbol{D} \\
\boldsymbol{D} Z_{i}^{\prime} & \boldsymbol{D}
\end{array}\right]\right)
$$




\section{Estimation in LME models}

The parameters in the LME models are generally estimated by maximum likelihood (ML) method or restricted maximum likelihood (REML) method. Under the distribution assumptions for random effects $b_{i}$ and random errors $\varepsilon_{i}(i=1, \ldots, N)$, the response variable for $i^{\text {th }}$ subject (i.e., $y_{i}$ ) has mean and variance as follows:

$$
\begin{gathered}
E\left(y_{i}\right)=X_{i} \beta, \\
\operatorname{Var}\left(\boldsymbol{y}_{i}\right)=\Sigma_{i}(\theta)=Z_{i} \boldsymbol{D} Z_{i}^{\prime}+\sigma^{2} I_{n_{i}} .
\end{gathered}
$$

In case $\Sigma_{i}(\theta)=\Sigma_{i}$ is known, the ML estimate of the fixed effects $\beta$ is:

$$
\hat{\beta}=\left\{\sum_{i=1}^{N}\left(X^{\prime}{ }_{i} \Sigma_{i}{ }^{-1} X_{i}\right)\right\}^{-1} \sum_{i=1}^{N}\left(X^{\prime}{ }_{i} \Sigma_{i}{ }^{-1} y_{i}\right) .
$$

However, the variance component parameters, i.e., the parameters in the variance of $b_{i}$ (say $\boldsymbol{D}$ ) and the variance $\varepsilon_{i}$ (say $\sigma^{2}$ ), are unknown and need to be estimated by ML method or REML method. Let us denote the variance component parameters as $\theta$, and their estimator as $\hat{\theta}$. Then the ML estimate of the fixed effects $\beta$ is estimated by the following equation (E 2.4), where $\Sigma_{i}$ in (E 2.3) is replaced by $\Sigma_{i}(\hat{\theta})$ :

$$
\hat{\beta}=\left\{\sum_{i=1}^{N}\left(X^{\prime}{ }_{i} \hat{\Sigma}_{i}^{-1} X_{i}\right)\right\}^{-1} \sum_{i=1}^{N}\left(X^{\prime}{ }_{i} \hat{\Sigma}_{i}^{-1} y_{i}\right) .
$$

Generally, the REML estimator for the variance component in $\Sigma_{i}$ is recommended because the REML estimate provides unbiased estimators for variance components [18]. In this thesis, we use REML method to obtain estimates of variance components in LME model (E 2.1) and then obtain the estimates of the fixed-effect parameters as in (E 2.4) [19]. 


\section{$\underline{\text { Statistical inference in LME models }}$}

Hypothesis tests are very important to examine whether certain covariates significantly contribute to the response variable, and whether certain parameters are the same, which may indicate no treatment effect. The associated statistic null hypothesis is $H_{0}: \lambda^{\prime} \beta=0$, and the alternative hypothesis is $H_{1}: \lambda^{\prime} \beta \neq 0$.

If the underlying assumptions for the random effects $b_{i}$ and within-subject errors $\varepsilon_{i}$ $(i=1 \ldots N)$ hold, the ML estimator of $\beta$, say $\hat{\beta}$, follows the following normal distribution:

$$
\hat{\beta} \sim N\left(\beta,\left\{\sum_{i=1}^{N}\left(X^{\prime}{ }_{i} \hat{\Sigma}_{i}^{-1} X_{i}\right)\right\}^{-1}\right) .
$$

The test statistic for testing $H_{0}: \lambda^{\prime} \beta=0$ versus $H_{1}: \lambda^{\prime} \beta \neq 0$ can be carried out using the Wald test statistic:

$$
Z=\frac{\lambda^{\prime} \hat{\beta}}{\sqrt{\lambda^{\prime} \widehat{\operatorname{Cov}}(\hat{\beta}) \lambda}} \sim N(0,1) .
$$

A special case for testing $H_{0}: \beta_{k}=0$ versus $H_{1}: \beta_{k} \neq 0$ can be carried out using the Wald test statistic:

$$
\frac{\hat{\beta}_{k}}{\sqrt{\operatorname{Var}\left(\hat{\beta}_{k}\right)}} \sim N(0,1) .
$$

An alternative to test for the hypothesis is the likelihood ratio test (LRT). The LRT for $H_{0}: \lambda^{\prime} \beta=0$ versus $H_{1}: \lambda^{\prime} \beta \neq 0$ is obtained by comparing the maximized loglikelihoods for two models: the full model versus the reduced model. The reduced model incorporates the constraint that $\lambda^{\prime} \beta=0$, while the full model does not (i.e., without the 
constraint $\lambda^{\prime} \beta=0$ ) [18]. The LRTs can also be used for hypothesis tests about the covariance components [18]. However, the REML method can be used to compare nested models only involving the reduction in covariance components but should not be used to compare nested regression models for reduction in fixed-effects terms [18].

\subsection{Nonlinear mixed-effects (NLME) models}

The general formula of the single level NLME models is proposed by Lindstrom and Bates [6]. At first level the $j^{\text {th }}$ observation on the $i^{\text {th }}$ subject is modeled as:

$$
y_{i j}=f\left(\phi_{i j}, v_{i j}\right)+\epsilon_{i j} \quad i=1, \ldots, N, \quad j=1, \ldots, n_{i}, \quad \epsilon_{i j} \sim N\left(0, \sigma^{2}\right)
$$

where $N$ is the number of subjects, $n_{i}$ is the number of observations on the $i^{\text {th }}$ subject, $f$ is a real-valued differentiable function of a subject specific parameter vector $\phi_{i j}$, and covariate vector $v_{i j}$, and $\epsilon_{i j}$ is a normally distributed within-subject error term. The function $f$ is nonlinear in at least one component of the group-specific parameter vector $\phi_{i j}$, which is modeled as:

$$
\phi_{i j}=A_{i j} \beta+B_{i j} b_{i}, \quad b_{i} \sim N(0, \Psi)
$$

where $\beta$ is a p-dimensional vector of fixed effects, and $b_{i}$ is a $q$-dimensional random effects vector associated with the $i^{\text {th }}$ subject with variance-covariance matrix $\Psi$. The matrices $A_{i j}$ and $B_{i j}$ are of appropriate dimensions and depend on the $i^{\text {th }}$ subject and possibly on the values of some covariates at the $j^{\text {th }}$ observation. Again here, $b_{i}$ and $\epsilon_{i j}=\left(\epsilon_{i 1}, \ldots, \epsilon_{i n_{i}}\right)^{T}$ are assumed to be normally distributed with mean zero and variance $D$ and $\Psi$, respectively. 


\section{Estimation in NLME models}

Different parameter estimations have been proposed in the NLME model [17]. In this thesis, we will describe methods based on the likelihood function. There are three methods for approximating the likelihood function in the NLME model; the LME approximation proposed by Lindstrom and Bates, the method of using Laplacian approximation to the likelihood function, and the method of using an adaptive Gaussian quadrature rule to improve the Laplacian approximation [17]. A REML method using the alternating algorithm was also proposed by Lindstrom and Bates [15]. Of these methods, the alternating algorithm of Lindstrom and Bates is used for estimation and inference in our nonlinear models. The estimation algorithm of Lindstrom and Bates for the singlelevel NLME model alternates two steps, a penalized nonlinear least square (PNLS) step and a linear mixed effects (LME) step.

In the PNLS step, the current estimate of $\Delta$ (the precision factor) is held fixed, and the conditional modes of the random effects $b_{i}$ and conditional estimates of the fixed effects $\beta$ are obtained by minimizing a penalized nonlinear least squares objective function:

$$
\sum_{i=1}^{M}\left\|y_{i}-f_{i}\left(\beta, b_{i}\right)\right\|^{2}+\left\|\Delta b_{i}\right\|^{2} .
$$

The LME step updates the estimate of $\Delta$ based on a first-order Taylor expansion of the model function $f$ around the current estimates of $\beta$ and the conditional modes of the random effects $b_{i}$, which will be denoted by $\hat{\beta}^{(w)}$ and $\hat{b}_{i}^{(w)}$, respectively. Letting 


$$
\begin{gathered}
\hat{X}_{i}^{(\omega)}=\left.\frac{\partial f_{i}}{\partial \beta^{T}}\right|_{\widehat{\beta}^{(\omega)}, \hat{b}_{i}^{(\omega)}}, \quad \hat{Z}_{i}^{(\omega)}=\left.\frac{\partial f_{i}}{\partial b^{T}}\right|_{\widehat{\beta}^{(\omega)}, \hat{b}_{i}^{(\omega)^{\prime}}} \\
\widehat{\omega}_{i}^{(\omega)}=y_{i}-f_{i}\left(\hat{\beta}^{(\omega)}, \hat{b}_{i}^{(\omega)}\right)+\hat{X}_{i}^{(\omega)} \hat{\beta}^{(\omega)}+\hat{Z}_{i}^{(\omega)} \hat{b}_{i}^{(\omega)},
\end{gathered}
$$

the appropriate log-likelihood function used to estimate $\Delta$ is:

$$
\begin{gathered}
\ell_{L M E}\left(\beta, \sigma^{2}, \Delta \mid y\right)=-\frac{N}{2} \log \left(2 \pi \sigma^{2}\right)-\frac{1}{2} \sum_{i=1}^{M}\left\{\log \left|\Sigma_{i}(\Delta)\right|\right. \\
+\sigma^{-2}\left[\widehat{\omega}_{i}^{(\omega)}-\hat{X}_{i}^{(\omega)} \beta\right]^{T} \Sigma_{i}^{-1}(\Delta)\left[\widehat{\omega}_{i}^{(\omega)}-\hat{X}_{i}^{(\omega)} \beta\right]
\end{gathered}
$$

where $\Sigma_{i}(\Delta)=I+\hat{Z}_{i}^{(\omega)} \Delta^{-1} \Delta^{-T} \hat{Z}_{i}^{(\omega)^{T}}$.

Lindstrom and Bates also proposed a REML estimation method for $\Delta$, which consists of replacing the log-likelihood in the LME step of the alternation algorithm by the logrestricted-likelihood. The algorithm alternates between the PNLS and LME steps until a convergence criterion is met. The algorithm has been implemented by Lindstrom and Bates [15] in R, which can be called by function nlme in R.

\section{Statistical Inference in NLME models}

Inference on the parameters of an NLME model estimated via the alternating algorithm is based on the LME approximation to the log-likelihood function. At the convergence, under the normality assumption for the random effects and within-subject errors, the estimator $\beta$ has the following distribution:

$$
\hat{\beta} \sim N\left(\beta, \sigma^{2}\left[\sum_{i=1}^{M} \hat{X}_{i}^{T} \Sigma_{i}^{-1} \hat{X}_{i}\right]^{-1}\right)
$$


where $\Sigma_{i}(\hat{\Delta})=I+\hat{Z}_{i}^{(\omega)} \hat{\Delta}^{-1} \hat{\Delta}^{-T} \hat{Z}_{i}^{(\omega)^{T}}, \quad \hat{X}_{i}^{(\omega)}=\left.\frac{\partial f_{i}}{\partial \beta^{T}}\right|_{\widehat{\beta}^{(\omega)}, \hat{b}_{i}^{(\omega)}}$, and $\quad \hat{Z}_{i}^{(\omega)}=\left.\frac{\partial f_{i}}{\partial b^{T}}\right|_{\widehat{\beta}^{(\omega)}, \hat{b}_{i}^{(\omega)}}$.

The test statistic for testing $H_{0}: \lambda^{\prime} \beta=0$ versus $H_{1}: \lambda^{\prime} \beta \neq 0$ can be carried out using the Wald test statistic:

$$
z=\frac{\lambda^{\prime} \hat{\beta}}{\sqrt{\lambda^{\prime} \widehat{\operatorname{Cov}}(\hat{\beta}) \lambda}} \sim N(0,1) .
$$

A special case for testing $H_{0}: \beta_{k}=0$ versus $H_{1}: \beta_{k} \neq 0$ can be carried out using the Wald test statistic:

$$
z=\frac{\hat{\beta}_{k}}{\sqrt{\operatorname{Var}\left(\hat{\beta}_{k}\right)}} \sim N(0,1)
$$

\subsection{Method of estimation of change point in two phase growth models}

Suppose the growth model contains two growth phases: $f_{1}\left(t ; \beta_{1}\right)$ at the first phase where $t \leq \gamma$ and $f_{2}\left(t ; \beta_{2}\right)$ at the second phase where $t \geq \gamma$. Let us denote $y(t)$ as the response variable at time, then $y(t)$ is expressed as:

$$
y(t)= \begin{cases}f_{1}\left(t ; \beta_{1}\right) & \text { if } t \leq \gamma \\ f_{2}\left(t ; \beta_{2}\right) & \text { if } t \geq \gamma .\end{cases}
$$

$\gamma$ is the unknown time where the growth curve from the first phase shifts to the secondary phase. $\gamma$ is called the change point [22]. $\beta_{1}$ and $\beta_{2}$ are unknown parameters in the phase 1 and phase 2 curves, respectively. The continuous change implies $f_{1}\left(\gamma ; \beta_{1}\right)=f_{2}\left(\gamma ; \beta_{2}\right)$. To search for the change point, the least squares estimate (LSE) is obtained. 
An algorithm for calculating a least squares estimate (LSE)

Let us denote $\left(t_{i}, y_{i}\right)(i=1, \ldots, n)$ as $n$ observations, ordered in such a way that $t_{1} \leq t_{2} \leq \cdots \leq t_{n}$. We assume that the unknown change point $\gamma$ belongs to a given interval $\Gamma=[\bar{\gamma}, \underline{\gamma}]$. For the change point $\gamma \in \Gamma$, there exists a natural number $m(\gamma)$ with $2 \leq m(\gamma) \leq n-1$ such that the first $m(\gamma)$ observations $\left(t_{i}, y_{i}\right), \quad(i=$ $1, \ldots, m(\gamma))$ belong to phase 1 and the last $(n-m(\gamma))$ observations $\left(t_{i}, y_{i}\right), \quad(i=$ $m(\gamma)+1, \ldots, n)$ belong to the phase 2. $m(\gamma)$ depends on the unknown change point $\gamma$ and $\gamma$ satisfies $t_{m(\gamma)} \leq \gamma \leq t_{m(\gamma)+1}$, where $m(\gamma)$ is called change index. The LSE for model parameters $\left(\beta_{1}, \beta_{2}, \gamma\right)$ is defined as the solution of minimizing the following sum of squares:

$$
\mathrm{S}\left(\beta_{1}, \beta_{2}, \gamma\right)=\sum_{j=1}^{m(\gamma)}\left(y\left(t_{i}\right)-f_{1}\left(t_{i} ; \beta_{1}\right)\right)^{2}+\sum_{i=m(\gamma)+1}^{n}\left(y\left(t_{i}\right)-f_{2}\left(t_{i} ; \beta_{2}\right)\right)^{2},
$$

under the restriction $f_{1}\left(\gamma ; \beta_{1}\right)=f_{2}\left(\gamma ; \beta_{2}\right)$.

The detailed descriptions for the estimation change point are found in Schulze [19].

\subsection{Model selection}

\section{Likelihood Ratio Test (LRT)}

The likelihood ratio test (LRT) can be used to compare nested models. The LRT statistic is obtained as:

$$
L R T=2\left(\log L_{\text {full }}-\log L_{\text {reduced }}\right)
$$


where $\log L_{f u l l}$ and $\log L_{\text {reduced }}$ are the maximized $\log$-likelihood values for the full and reduced models, respectively. $L R T$ is compared to the chi-square distribution with degrees of freedom being the number of additional parameters in the full model versus the reduced model. In this thesis, we start from a full model. Then we apply the LRT and the following Akaike Information Criterion (AIC) to remove the least significant term using a backward procedure, and obtain a parsimonious model for the final interpretation.

\section{Akaike Information Criterion (AIC) and Baysian Information Criterion (BIC)}

Akaike Information Criterion (AIC) is used to compare models that are not nested and defined for a given model as [20]:

$$
\mathrm{AIC}=-2 \log L+2 p,
$$

where $p$ is the number of parameters in the model, and the $L$ is the likelihood function at the estimated parameters.

The model with the smallest AIC value is preferred. The adjustment in AIC to the deviance is often called the penalty for using additional parameters in model fitting.

Another criterion for model selection is the Baysian Information Criterion (BIC) [21]. This criterion is defined as:

$$
\mathrm{BIC}=-2 \log L+p \log N,
$$

where $N$ is the number of observations, $p$ is the number of parameters in the model, and the $L$ is the likelihood function at the estimated parameters.

As in AIC, the model with the lowest BIC value is preferred. The detailed descriptions 
about model selection are referred to Donald and Robert [3]. 


\section{CHAPTER 3}

\section{Applications}

In this section, we applied mixed-effects models and change point model to investigate the contribution of Glutathione-S-transferase P (GSTP) gene on the recovery of post-ischemic cardiac function. As a member of a large family of GSTs [23], GSTP is a major cardiovascular GST isoform and accounts for $20-50 \%$ of the total GST activity in mouse aorta [24] and heart [25]. To examine the impact of GSTP on postischemic cardiac function, the experiments were carried out in Dr. Conklin's laboratory. Cardiac function was assessed by measurements of heart rate, coronary flow, and left ventricle (LV) developed pressure. To examine the effect of the deletion of the GSTP gene in myocardial ischemia-reperfusion ( $\mathrm{I} / \mathrm{R})$ injury, the cardiac function of knockout $(\mathrm{KO})$ mice was compared with wild-type (WT) mice. Each aspect of the cardiac function data was collected during three time periods: pre-ischemia, ischemia, and reperfusion periods. We developed piecewise nonlinear mixed-effects model to describe the different aspects of the cardiac function, and applied the nonlinear mixed-effects models, change point model, and model reduction techniques to obtain a parsimonious model for the final interpretation. We performed hypothesis tests to examine the impact of deletion of GSTP for different aspects of cardiac function including testing whether cardiac function 
including testing whether cardiac performance eventually recovers during reperfusion period.

\subsection{Experimental data and graphical presentation}

\section{Experimental Data}

Data were obtained from Glutathione-S-transferase P (GSTP) gene KO mice and WT mice hearts. Three different datasets were obtained for three response variables: namely, measurements of heart rate, coronary flow, and LV developed pressure. Cardiac function was measured in hearts exposed to ischemia/reperfusion injury by subjecting isolated hearts to 5 minutes of pre-ischemia, 30 minutes of global ischemia and 45 minutes of reperfusion in six KO and seven WT hearts. The measurements of each one of the three response variables are presented in Figures 1, 2, and 3, respectively.

\section{Graphical presentation}

Figures 1 to 3 show how the heart rate, coronary flow, and LV developed pressure change over different time periods for GSTP KO mice and WT mice. As illustrated in Figures 1 to 3, there was no significant difference between WT mice and KO mice during pre-ischemia for each one of the three endpoints. Figure 1 indicates that during reperfusion, heart rate returned to the pre-ischemic range in WT mice but was greater than the pre-ischemic range in KO mice. Figure 2 indicates that the recovery of coronary flow was better in WT mice than $\mathrm{KO}$ mice during reperfusion. Figure 3 shows that LV developed pressure started to increase after a few minutes of 
perfusion in both WT and $\mathrm{KO}$ mice and recovered to a much greater extent in WT mice than $\mathrm{KO}$ mice. Figure 3 also indicates that ischemia/reperfusion injury significantly decreased LV developed pressure.

\subsection{Nonlinear model and statistical analysis for heart rate}

The measurements of heart rate over time in different periods for each individual are presented in Figure 1. We first carry out the analysis for each one of the three time period separately, then we build an integrated model to describe the heart rate over time for the entire experiment. Hypothesis tests are carried out to examine whether the heart rate in WT mice is significantly different from that in GSTP KO mice, and whether the heart rate during reperfusion recovers to the level of pre-ischemia.

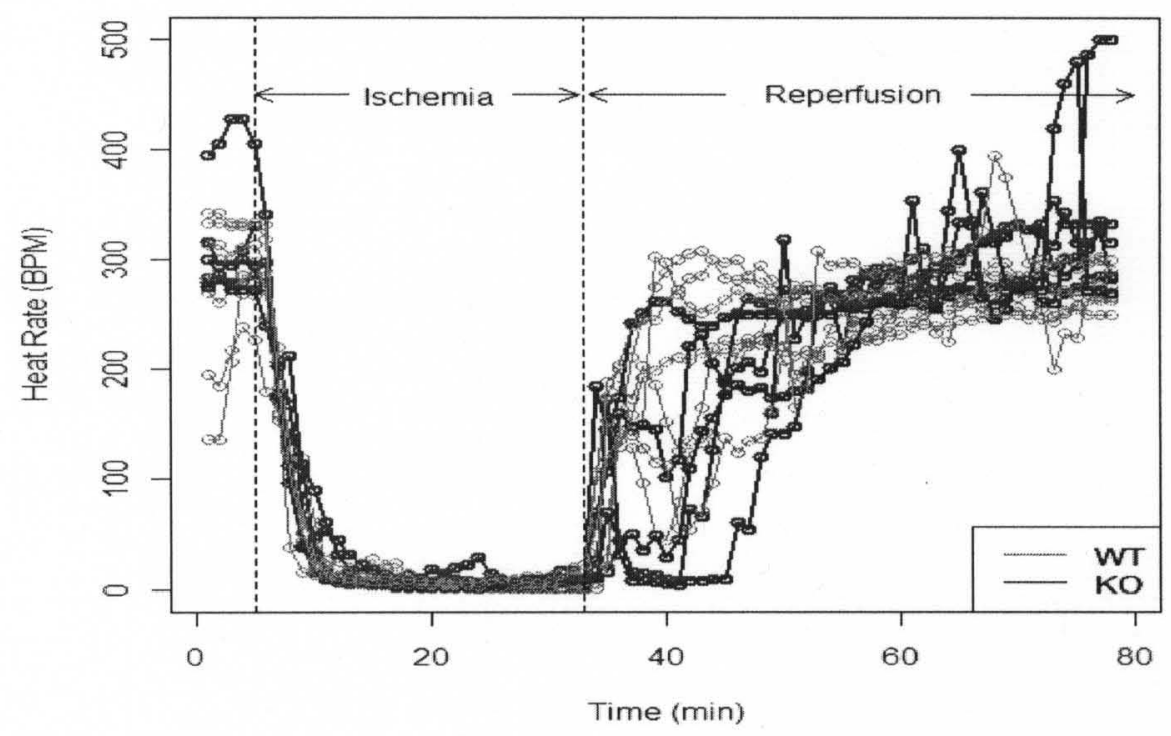

Figure 1. Individual profile for heart rate. 


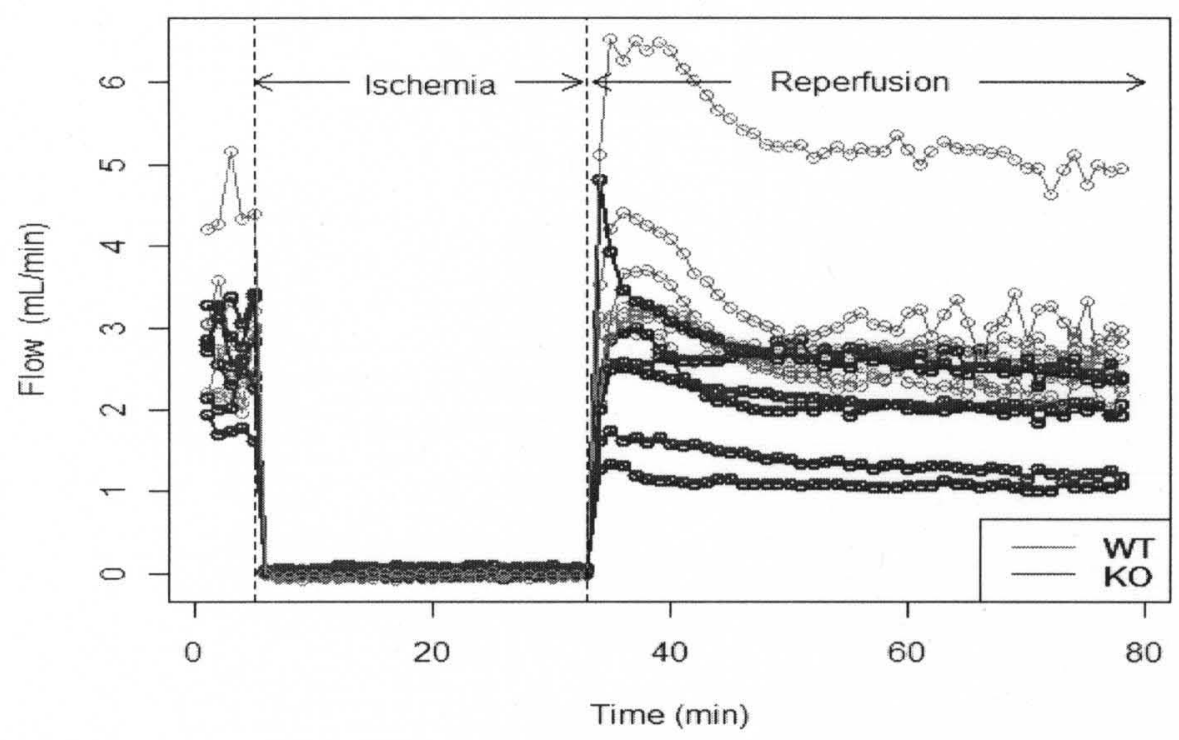

Figure 2. Individual profile for coronary flow.

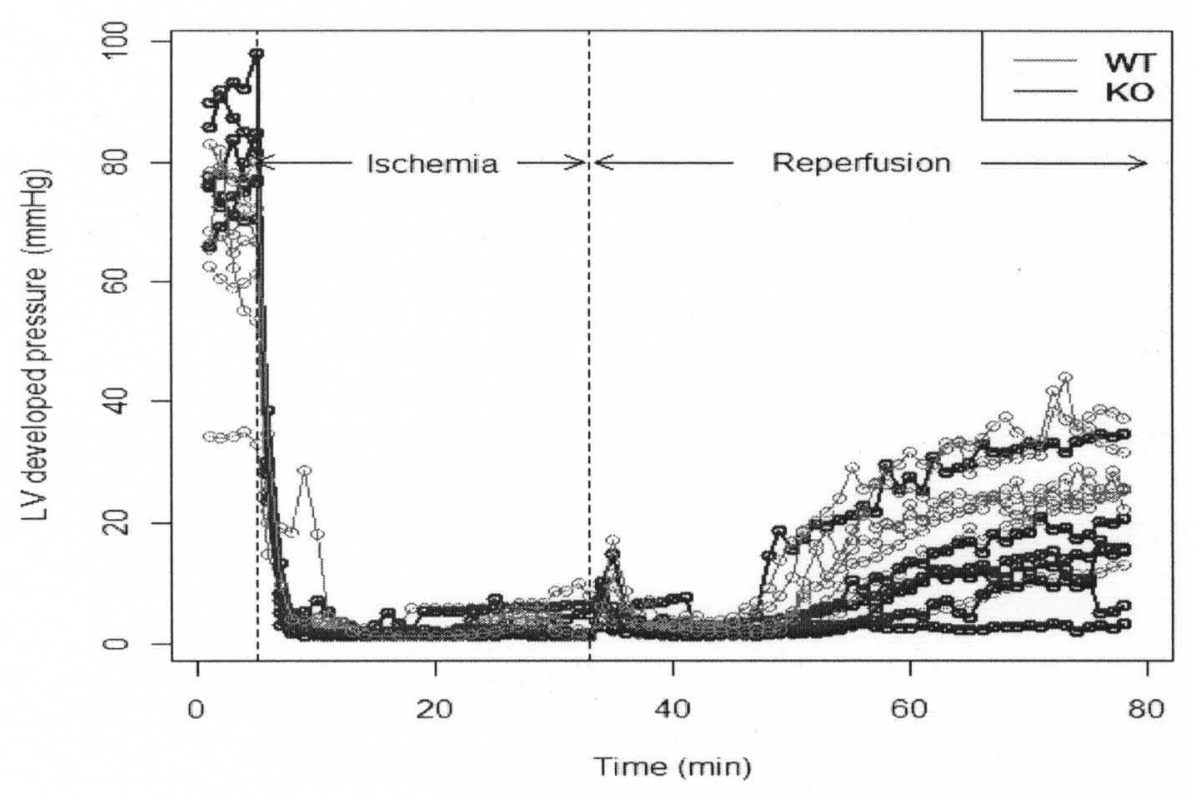

Figure 3. Individual profile for LV developed pressure. 


\section{Model and analysis of heart rate during pre-ischemia}

To examine the heart rate during pre-ischemia, we started with a full linear mixedeffects model with the following form:

$$
y_{i j}=\beta_{0}+\beta_{1} t_{i j}+\beta_{2} \text { type }_{i}+\beta_{3} t_{i j} \text { type }_{i}+b_{0 i}+b_{1 i} t_{i j}+\varepsilon_{i j},
$$

where $y_{i j}$ is the measurement of heart rate for subject $i$ at time $t_{i j}(i=1, \ldots, N)$,

$$
\begin{aligned}
{\left[\begin{array}{l}
b_{0 i} \\
b_{1 i}
\end{array}\right] } & =N\left(\left(\begin{array}{l}
0 \\
0
\end{array}\right),\left(\begin{array}{cr}
\sigma_{0}^{2} & \rho \sigma_{0} \sigma_{1} \\
\rho \sigma_{0} \sigma_{1} & \sigma_{1}^{2}
\end{array}\right)\right), \quad \varepsilon_{i j} \sim N\left(0, \sigma^{2}\right), \\
\text { type }_{i} & = \begin{cases}1 & \text { if mouse } i \text { is knockout } \\
0 & \text { if mouse } i \text { is wild - type. }\end{cases}
\end{aligned}
$$

The fixed effect parameters are $\beta_{0}, \beta_{1}, \beta_{2}$, and $\beta_{3} . \beta_{0}$ is the mean of the heart rate of WT mice at the starting point (i.e., $t_{i j}=0$ ); $\beta_{1}$ describes the change rate of the heart rate for WT mice during pre-ishemia; $\beta_{2}$ is the mean difference of the heart rates of the KO mice versus WT mice at the starting time of the experiment; $\beta_{3}$ is the slope difference of the heart rates between KO mice versus WT mice. The random effect $b_{0 i}(i=1, \ldots, N)$ describes the deviation of the heart rate of $i^{\text {th }}$ subject away from its group average at time $t_{i j}=0$, and $b_{1 i}(i=1, \ldots, N)$ is the deviation of the slopes for $i^{t h}$ subject away from its group-level slope.

We start from the full model (E 3.1), and then the least significant term was removed using the Wald test and AIC. The process was repeated until the AIC reaches its minimum. As a result, we could remove time and interaction terms in the fixed effects but could not remove the two random effects. Type in fixed effects (say, $\beta_{2}$ ) was not significant. However, we kept this parameter for testing whether or not heart rate is significantly different between the pre-ischemia and reperfusion period for both WT mice 


\section{TABLE 1}

Estimates of fixed-effects and variance components for linear mixed-effects model for heart rate during pre-ischemia

\begin{tabular}{crcrr}
\hline Parameter & Estimate & 95\% C.I. & $Z$ & P-value \\
\hline $\boldsymbol{\beta}_{\mathbf{0}}$ & 295.61 & {$[260.67,330.55]$} & 18.17 & 0.00 \\
$\boldsymbol{\beta}_{\mathbf{2}}$ & 14.72 & {$[-41.69,71.13]$} & 0.61 & 0.55 \\
$\mathbf{\sigma}_{\mathbf{0}}$ & 78.73 & {$[51.87,119.49]$} & & \\
$\boldsymbol{\sigma}_{\mathbf{1}}$ & 12.49 & {$[8.23,18.95]$} & & \\
$\boldsymbol{\rho}$ & -0.82 & {$[-0.94,-0.49]$} & & \\
$\boldsymbol{\sigma}$ & 11.45 & {$[9.17,14.30]$} & & \\
\hline
\end{tabular}

and KO mice. Table 1 shows the estimates of the fixed-effects and the variance components for the final model for pre-ischemia period.

The linear mixed-effects model (E 3.1) was applied to pre-ischemia, ischemia, and reperfusion periods, respectively, for each aspect of cardiac function. The results are presented in Appendix A.

\section{Model and analysis of heart rate during ischemia}

Figure 1 shows that the pattern of heart rate change over time during ischemia is not linear, and resembles exponential decline. Therefore, nonlinear mixed-effects model was considered for accurate approximation of the response variable. We used exponential function of the form $C_{1} \exp \left(-C_{2}\left(t_{i j}-T_{1}\right)_{+}\right)$to describe the dynamic change of the heart rate during ischemia, where $C_{1}$ describes the heart rate at the beginning of ischemia, $C_{2}(>0)$ describes the exponential decline rate, $\left(t-T_{1}\right)+$ takes $t-T_{1}$ if $t-T_{1}>0$ and 0 otherwise, where $T_{1}$ is a known constant, representing the time to start ischemia injury. We fit a nonlinear mixed-effects model of the following form for heart rate during ischemia: 


$$
y_{i j}=C_{1 i} \exp \left(-C_{2 i}\left(t_{i j}-T_{1}\right)_{+}\right)+\epsilon_{i j}
$$

where

$$
\begin{gathered}
C_{k i}=\beta_{1 k}+\beta_{2 k} \text { type }_{i}+b_{k i} \quad k=1,2, \\
{\left[\begin{array}{l}
b_{1 i} \\
b_{2 i}
\end{array}\right]=N\left(\left(\begin{array}{l}
0 \\
0
\end{array}\right),\left(\begin{array}{ll}
\sigma_{1}^{2} & \rho \sigma_{1} \sigma_{2} \\
\rho \sigma_{1} \sigma_{2} & \sigma_{2}^{2}
\end{array}\right)\right), \varepsilon_{i j} \sim N\left(0, \sigma^{2}\right),} \\
\text { type }_{i}=\left\{\begin{array}{cc}
1 & \text { if mouse } i \text { is knockout } \\
0 & \text { if mouse } i \text { is wild- type. }
\end{array}\right.
\end{gathered}
$$

$C_{1 i}$ is the value of $y_{i j}$ at $t_{i j}=T_{1}$ and $C_{2 i}$ is the constant exponential decline rate.

The fixed effect $\beta_{11}$ represents the mean of the heart rate for WT mice at the beginning of ischemia, and $\beta_{11}+\beta_{21}$ represents the mean of heart rate for $\mathrm{KO}$ mice at the beginning of ischemia. The random effect $b_{1 i}(i=1, \ldots, N)$ describes the deviation of the heart rate for $i^{\text {th }}$ subject away from its group mean. Similarly, $\beta_{12}$ and $\beta_{12}+\beta_{22}$ represent the exponential decline rate for WT mice and KO mice, respectively, and $b_{2 i}$ describes the deviation of the exponential decline rate for $i^{t h}$ subject from group-level decline rate.

Nonlinear mixed-effects model (E 3.2) was fitted to the ischemia data and the results are given in Table 2. From Table 2, it is clear that there is no significant difference for the heart rate at the beginning of ischemia between the two types of mice. However, the exponential decline rate for WT mice is significantly larger than for KO mice at significant level 0.1 . The fitted heart rates at group-level and the predicted heart rates at subject-level, as well as the observed heart rates are presented in Figure 4. As can be seen in Figure 4, the subject-level predictions closely matched the observed heart rates during ischemia indicating the goodness of fit of the model.

Model and analysis of heart rate during reperfusion 
TABLE 2

Estimates of fixed-effects and variance components for nonlinear mixed-effects model (E 3.2) for heart rate during ischemia

\begin{tabular}{crrcrr}
\hline Parameter & Estimate & S.E. & $95 \%$ C.I & $Z$ & P-value \\
\hline $\boldsymbol{\beta}_{\mathbf{1 1}}$ & 458.23 & 26.46 & {$[406.48,509.97]$} & 17.32 & 0.00 \\
$\boldsymbol{\beta}_{\mathbf{2 1}}$ & -15.59 & 40.34 & {$[-94.49,63.31]$} & -0.39 & 0.70 \\
$\boldsymbol{\beta}_{\mathbf{1 2}}$ & 0.50 & 0.03 & {$[0.45,0.55]$} & 18.50 & 0.00 \\
$\boldsymbol{\beta}_{\mathbf{2 2}}$ & -0.07 & 0.04 & {$[-0.15,0.00]$} & -1.83 & 0.07 \\
$\boldsymbol{\sigma}_{\mathbf{1}}$ & 62.68 & & {$[38.02,103.35]$} & & \\
$\boldsymbol{\sigma}_{\mathbf{2}}$ & 0.06 & & {$[0.04,0.10]$} & & \\
$\boldsymbol{\rho}$ & 0.5 & & {$[-0.17,0.85]$} & & \\
$\boldsymbol{\sigma}$ & 12.02 & & {$[11.11,13.00]$} & & \\
\hline
\end{tabular}

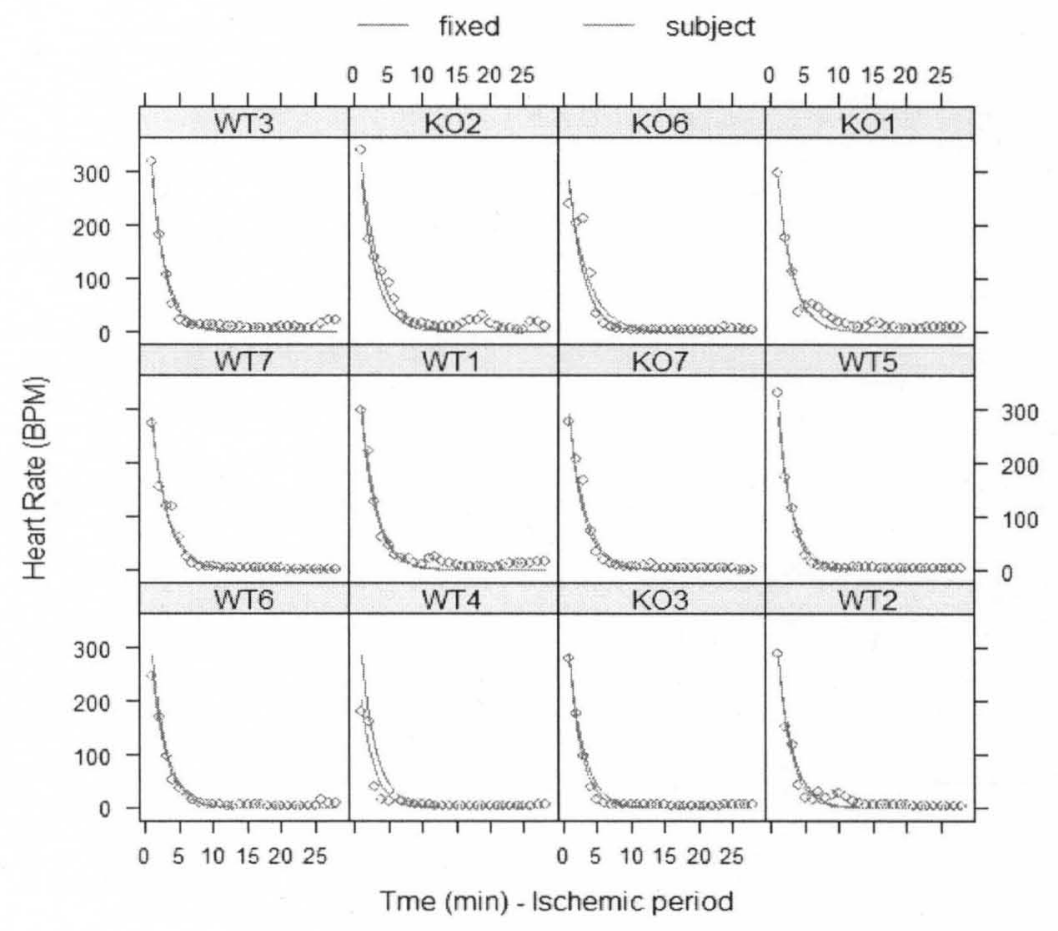

Figure 4. Plots of the fitted heart rates at group-level and the predicted heart rates at subject-level, as well as the observed heart rates (circles) versus time during ischemia.

Figure 1 shows that the pattern of heart rate during reperfusion is nonlinear.

The heart rate during reperfusion increases and reaches a plateau. The nonlinear of the 
the form $f\left(t ; C_{3}, C_{4}\right)=C_{3}\left[1-\exp \left(-C_{4}\left(t-T_{2}\right)_{+}\right)\right]$describes this scenario, where $C_{3}$ is the plateau of heart rate when the time for reperfusion goes to large, and $C_{4}$ is related to the increasing rate of heart rate during reperfusion. The nonlinear mixed-effects model to describe the heart rate during reperfusion can be expressed as:

$$
y_{i j}=C_{3 i}\left[1-\exp \left(-C_{4 i}\left(t_{i j}-T_{2}\right)_{+}\right)\right]+\epsilon_{i j}
$$

where

$$
\begin{gathered}
C_{k i}=\beta_{1 k}+\beta_{2 k} \text { type }_{i}+b_{k i} \quad k=3,4, \\
{\left[\begin{array}{l}
b_{3 i} \\
b_{4 i}
\end{array}\right]=N\left(\left(\begin{array}{l}
0 \\
0
\end{array}\right),\left(\begin{array}{lr}
\sigma_{3}^{2} & \rho \sigma_{3} \sigma_{4} \\
\rho \sigma_{3} \sigma_{4} & \sigma_{4}^{2}
\end{array}\right)\right), \quad \varepsilon_{i j} \sim N\left(0, \sigma^{2}\right),} \\
\text { type }_{i}=\left\{\begin{array}{rr}
1 & \text { if mouse } i \text { is knockout } \\
0 & \text { if mouse } i \text { is wild-type } .
\end{array}\right.
\end{gathered}
$$

$C_{3 i}$ is the asymptote as $t_{i j} \rightarrow \infty$ and $C_{4 i}$ is the constant exponential increasing rate.

Model (E 3.3) can be fitted by using the function SSasymOrig in R [17]. Figure 5 shows that the fitted heart rates at group-level and the prediction heart rates at subjectlevel. Nonlinear mixed-effects model (E 3.3) was fitted and the results are given in Table 3. An overall assessment of the quality of the nonlinear mixed-effects model (E 3.3) is shown in Figure 5. As can be seen in Figure 5, subject-level predictions closely matched the observed heart rates during reperfusion indicating the goodness of fit of the model.

\section{An integrated mixed-effects model for heart rate during the entire experiment}

The preliminary analyses of heart rate data indicate that linear mixed-effects model is appropriate to describe the heart rate during pre-ischemia and nonlinear 


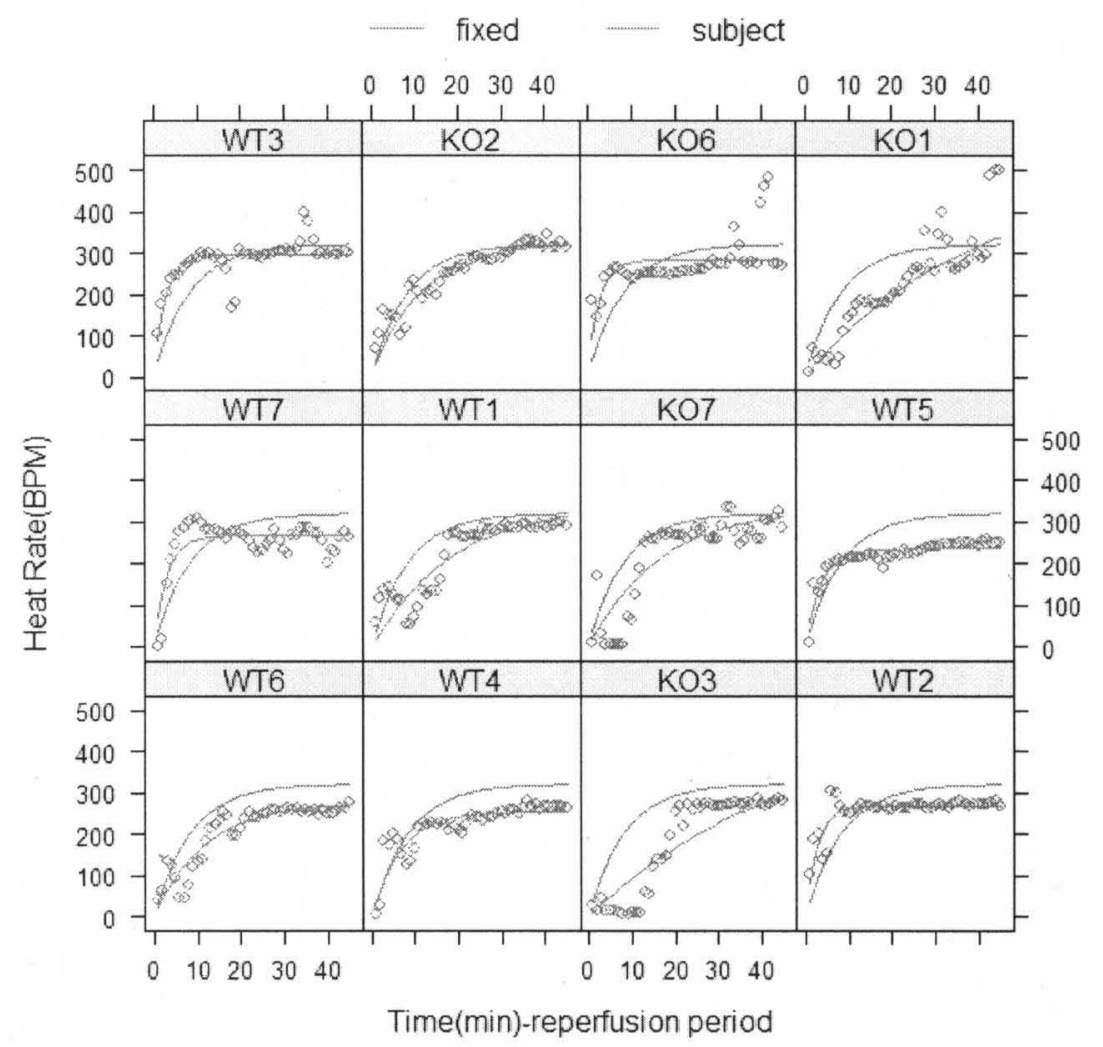

Figure 5. Plots of the fitted heart rates at group-level and the predicted heart rates at subject-level, as well as the observed heart rates versus time during reperfusion.

TABLE 3

Estimates of fixed-effects and variance components for nonlinear mixed-effects model (E 3.3) for heart rate during reperfusion

\begin{tabular}{crrcrr}
\hline Parameter & Estimate & S.E. & $95 \%$ C.I. & $Z$ & P-value \\
\hline $\boldsymbol{\beta}_{13}$ & 276.49 & 23.68 & {$[230.14,322.85]$} & 11.67 & 0.00 \\
$\boldsymbol{\beta}_{\mathbf{2 3}}$ & 107.35 & 39.81 & {$[29.43,185.26]$} & 2.70 & 0.01 \\
$\boldsymbol{\beta}_{14}$ & -1.66 & 0.33 & {$[-2.31,-1.01]$} & -4.97 & 0.00 \\
$\boldsymbol{\beta}_{\mathbf{2 4}}$ & -1.09 & 0.52 & {$[-2.10,-0.08]$} & -2.11 & 0.04 \\
$\boldsymbol{\sigma}_{\mathbf{3}}$ & 61.39 & & {$[21.02,179.29]$} & & \\
$\boldsymbol{\sigma}_{\mathbf{4}}$ & 0.86 & & {$[0.54,1.38]$} & & \\
$\boldsymbol{\rho}$ & -0.77 & & {$[-0.93,-0.34]$} & & \\
$\boldsymbol{\sigma}$ & 38.62 & & {$[36.29,41.10]$} & & \\
\hline
\end{tabular}


mixed-effects models are preferred to describe the heart rate for ischemia and reperfusion.

An integrated mixed-effects model for heart rate is expressed as:

$$
y_{i j}=\left\{\begin{array}{cl}
C_{1 i} & 0 \leq t_{i j} \leq 5 \\
C_{1 i} \exp \left(-C_{2 i}\left(t_{i j}-5\right)_{+}\right) & 5<t_{i j} \leq 33 \\
C_{3 i}\left[1-\exp \left(-C_{4 i}\left(t_{i j}-33\right)_{+}\right)\right] & 33<t_{i j} \leq 78
\end{array}\right.
$$

where

$$
\begin{aligned}
C_{k i} & =\beta_{1 k}+\beta_{2 k} \text { type }_{i}+b_{k i}, \quad k=1, \ldots, 4, \\
\text { type }_{i} & =\left\{\begin{array}{cc}
1 & \text { if mouse } i \text { is knockout } \\
0 & \text { if mouse } i \text { is wild-type } .
\end{array}\right.
\end{aligned}
$$

The interpretations of the parameters are exactly the same as those in the separated models. We start from the full model (E 3.4) with general variancecovariance on random effects. The approximate $95 \%$ confidence intervals on variancecovariance components for random effects indicate diagonal variance-covariance structure which covariances between the random effects are zero. To determine which variance-covariance structure is needed for random effects, AIC and LRT are used. As a result, the simpler variance-covariance structure for random effects (say, diagonal variance-covariance) was preferred. Table 4 shows the estimates of fixed-effects and the variance components for the integrated mixed-effects model (E 3.4) with diagonal variance-covariance structure on random effects for the entire experimental data. Figure 6 shows the plot of the standardized residuals versus the subject-level predicted values, which shows no evidence for the violation of the homogeneity of variance. An overall assessment of the integrated model (E 3.4) is provided by Figure 7, which indicates 


\section{TABLE 4}

Estimates of fixed-effects and variance components for the integrated mixed-effects model (E 3.4) for heart rate

\begin{tabular}{rrrcrr}
\hline Parameter & Estimate & S.E. & $95 \%$ C.I. & $\mathrm{Z}$ & P-value \\
\hline $\boldsymbol{\beta}_{\mathbf{1 1}}$ & 289.69 & 18.11 & {$[254.30,325.09]$} & 15.99 & 0.00 \\
$\boldsymbol{\beta}_{\mathbf{2 1}}$ & 27.59 & 28.06 & {$[-27.23,82.42]$} & 0.98 & 0.33 \\
$\boldsymbol{\beta}_{\mathbf{1 2}}$ & -1.10 & 0.05 & {$[-1.20,-1.00]$} & -21.55 & 0.00 \\
$\boldsymbol{\beta}_{\mathbf{2 2}}$ & -0.06 & 0.07 & {$[-0.21,0.08]$} & -0.84 & 0.49 \\
$\boldsymbol{\beta}_{\mathbf{1 3}}$ & 275.85 & 24.44 & {$[228.08,323.62]$} & 11.28 & 0.00 \\
$\boldsymbol{\beta}_{\mathbf{2 3}}$ & 110.79 & 41.86 & {$[28.99,192.59]$} & 2.65 & 0.01 \\
$\boldsymbol{\beta}_{\mathbf{1 4}}$ & -1.64 & 0.34 & {$[-2.30,-0.98]$} & -4.86 & 0.00 \\
$\boldsymbol{\beta}_{\mathbf{2 4}}$ & -1.12 & 0.53 & {$[-2.15,-0.09]$} & -2.14 & 0.03 \\
$\boldsymbol{\sigma}_{\mathbf{1}}$ & 45.65 & & & & \\
$\boldsymbol{\sigma}_{\mathbf{2}}$ & 0.00 & & & & \\
$\boldsymbol{\sigma}_{\mathbf{3}}$ & 63.71 & & & & \\
$\boldsymbol{\sigma}_{\mathbf{4}}$ & 0.88 & & & & \\
$\boldsymbol{\sigma}$ & 32.18 & & & & \\
\hline
\end{tabular}

that the proposed model fit this experimental data very well. Figure 8 shows that the recovery of heart rate in WT mice is more stable than in $\mathrm{KO}$ mice during reperfusion. Rigorous statistic tests and inference are provided in the following subsection for the entire experiment.

\section{$\underline{\text { Statistical test and inference for heart rate }}$}

To test whether the heart rate during reperfusion recovers to the level at pre-ischemia for WT mice, the underlying null hypothesis is $H_{0}: \beta_{11}-\beta_{13}=0$ and the alternative hypothesis is $H_{1}: \beta_{11}-\beta_{13} \neq 0$.

The Wald test statistic is:

$$
z=\frac{\lambda^{\prime} \hat{\beta}}{\sqrt{\lambda^{\prime} \widehat{\operatorname{Cov}}(\hat{\beta}) \lambda}}=0.389
$$




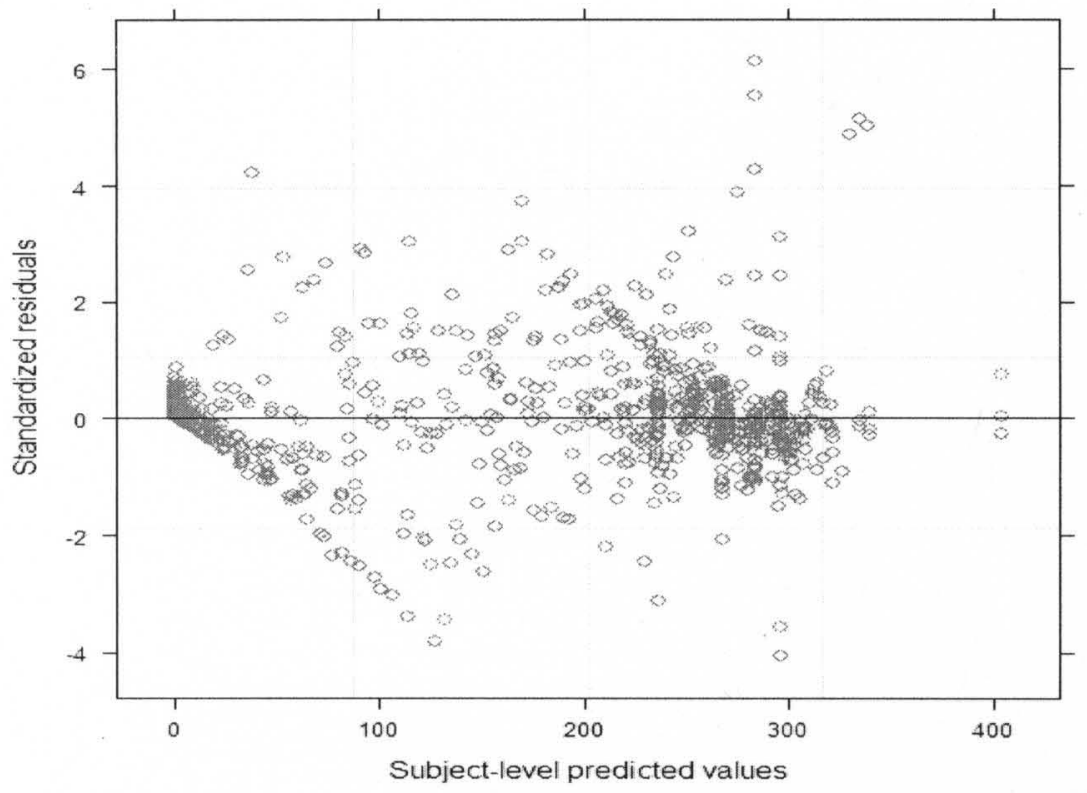

Figure 6. Diagnostic plot: Standardized residuals versus subject-level fitted heart rates.

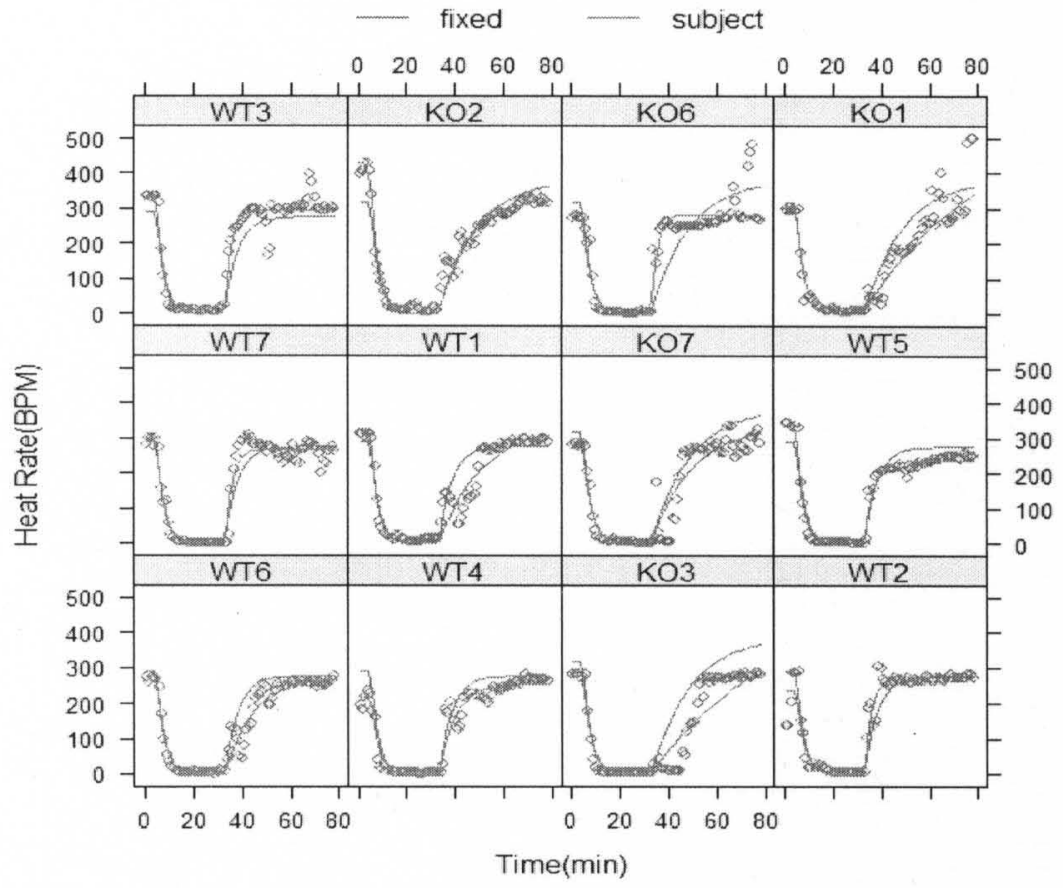

Figure 7. Plots of the fitted heart rates at group-level and the predicted heart rates at subject-level, as well as observed heart rates (circles) versus time for the entire experimental data. 


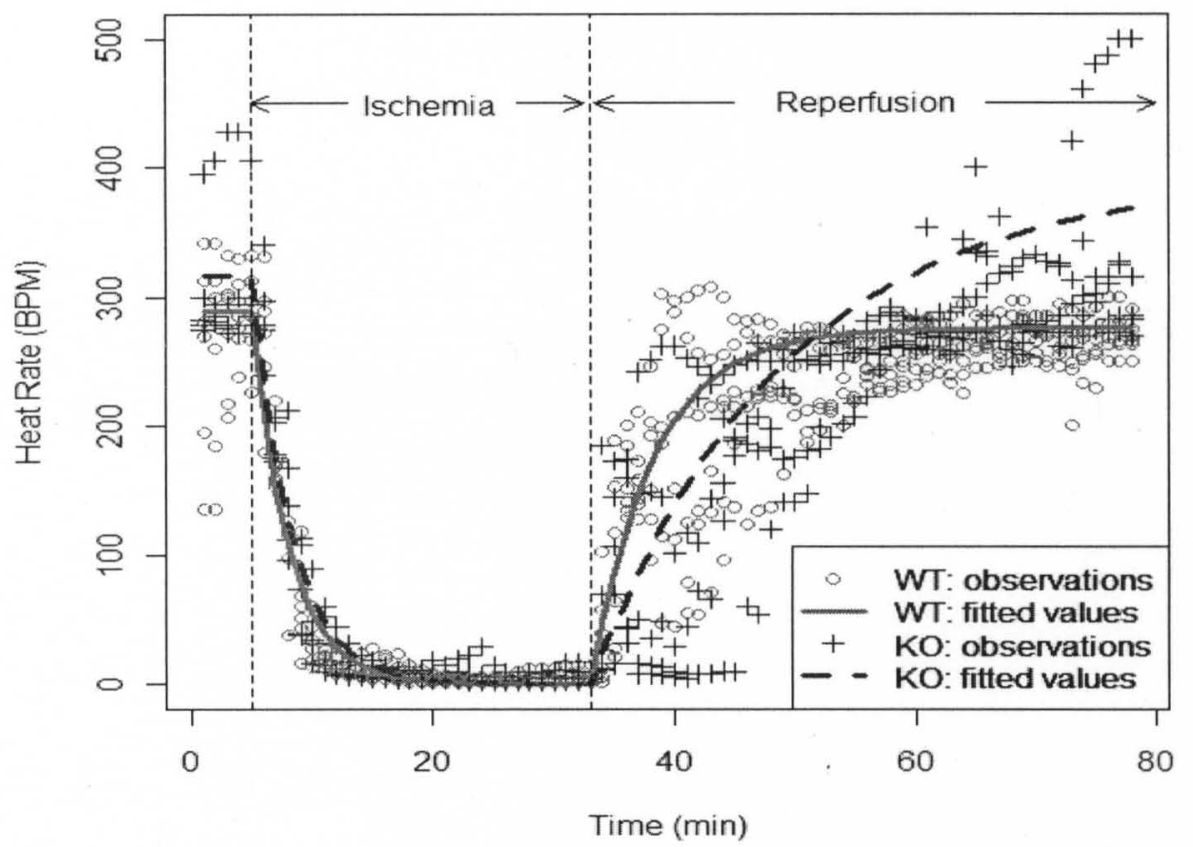

Figure 8. Fitted and observed heart rates versus time.

where $\lambda^{\prime}=(1,0,0,0,-1,0,0,0)$.

$P$-value is $2 P(z \geq 0.389)=0.697$, which indicates that we cannot reject $H_{0}$ at the $5 \%$ significance level. Therefore, we conclude that heart rate for WT mice during reperfusion recovered to the level at pre-ischemia.

To test whether the heart rate during reperfusion recovers to the level at pre-ischemia for $\mathrm{KO}$ mice, the underlying null hypothesis is $H_{0}: \beta_{11}+\beta_{21}-\beta_{13}-\beta_{23}=0$ and the alternative hypothesis is $H_{1}: \beta_{11}+\beta_{21}-\beta_{13}-\beta_{23} \neq 0$.

The Wald test statistic is:

$$
z=\frac{\lambda^{\prime} \hat{\beta}}{\sqrt{\lambda^{\prime} \widehat{\operatorname{Cov}}(\hat{\beta}) \lambda}}=-1.772,
$$


where $\lambda^{\prime}=(1,1,0,0,-1,-1,0,0)$ and $P$-value is $2 P(\mathrm{z} \geq|-1.772|)=0.077$.

Since $P$-value is 0.077 , we cannot reject $H_{0}$ at the $5 \%$ significance level. Therefore, we conclude that heart rate for $\mathrm{KO}$ mice during reperfusion recovered to the level at preischemia.

\subsection{Nonlinear model and statistical analysis for coronary flow}

An integrated mixed-effects model for coronary flow during the entire experiment

The preliminary analysis of coronary flow data during reperfusion indicated that the change in coronary flow was significantly different in KO mice compared with WT mice. In addition, linear mixed-effects models are appropriate to describe coronary flow during pre-ischemia and ischemia and nonlinear mixed-effects model is preferred to describe coronary flow during reperfusion period. The integrated mixed-effects model for coronary flow is expressed as:

$$
y_{i j}=\left\{\begin{array}{cc}
C_{1 i} & 0 \leq t_{i j} \leq 5 \\
C_{2 i} & 5<t_{i j} \leq 33 \\
C_{3 i}+\left(C_{4 i}-C_{3 i}\right) \exp \left(-C_{5 i}\left(t_{i j}-34\right)_{+}\right)-C_{7 i}\left[\exp \left(-C_{5 i}\left(t_{i j}-34\right)_{+}\right)-\exp \left(-C_{6 i}\left(t_{i j}-34\right)_{+}\right]\right. & 33<t_{i j} \leq 78
\end{array}\right.
$$

where

$$
\begin{aligned}
C_{k i} & =\beta_{1 k}+\beta_{2 k} t_{y p e_{i}}+b_{k i}, \quad k=1, \ldots, 7, \\
\text { type }_{i} & =\left\{\begin{array}{cc}
1 & \text { if mouse } i \text { is knockout } \\
0 & \text { if mouse } i \text { is wild - type }
\end{array}\right.
\end{aligned}
$$

$C_{3 i}$ is the asymptote as $t_{i j} \rightarrow \infty, C_{4 i}$ is the coronary flow immediately after ischemia at 
$t_{i j}=34$ and $C_{5 i}$ and $C_{6 i}$ are non-negative values and the logarithm of the rate constants. $\epsilon_{i j}$ is a normally distributed within-subject random errors. $b_{k i}(k=1, \ldots, 6)$ are random effect associated with $i^{\text {th }}$ subject. The fixed effect $\beta_{11}$ and $\beta_{11}+\beta_{21}$ are the mean of the coronary flows for WT mice and KO mice during pre-ischemia, respectively; $\beta_{12}$ and $\beta_{12}+\beta_{22}$ are the mean of coronary flows for WT mice and KO mice during ischemia, respectively; $\beta_{13}$ and $\beta_{13}+\beta_{23}$ represent the mean of coronary flows for WT mice and KO mice when the time for reperfusion goes to large, respectively; $\beta_{14}$ and $\beta_{14}+\beta_{24}$ are the mean coronary flows of WT mice and KO mice at the beginning of reperfusion; The random effect $b_{k i}(i=1, \ldots, N)$ describes the deviation of coronary flow for $i^{\text {th }}$ subject away from its group mean.

The integrated model (E 3.5) was fitted to the entire experimental data and the analysis results are given in Table 5 for estimates for fixed effects and Table 6 for estimates for variance components. Figure 9 shows the plot of the standardized residuals versus subject-level predicted values, which does not indicate any systematic deviation from the homogeneity of variance. An overall assessment of the integrated mixed-effects model (E 3.5) is shown in Figure 10. Both the fitted coronary flows at group-level and the predicted coronary flows at subject-level are displayed to show how individual effects are accounted for in the integrated mixed-effects model. As can be seen in Figure 10, the subject-level predictions matched the observed coronary flows very well for the entire experimental data indicating the goodness of fit of the model. Figure 11 shows that the recovery of coronary flow in WT is faster than that in KO mice during reperfusion. Rigorous statistical test and inference are provided in the following subsection. 
Statistical test and inference for coronary flow

To test whether the coronary flow during reperfusion recovers to the level of preischemia for WT, the underlying null hypothesis is $H_{0}: \beta_{11}-\beta_{13}=0$ and the alternative hypothesis is $H_{1}: \beta_{11}-\beta_{13} \neq 0$.

The Wald test statistic is:

$$
z=\frac{\lambda^{\prime} \hat{\beta}}{\sqrt{\lambda^{\prime} \widehat{\operatorname{Cov}}(\hat{\beta}) \lambda}}=-0.346,
$$

where $\lambda^{\prime}=(1,0,0,0,-1,0,0,0,0,0,0,0,0,0)$, and $P$-value is $2 P(z \geq|-0.346|)=0.730$.

Because $P$-value is 0.730 , we cannot reject $H_{0}$ at the $5 \%$ significance level. Therefore, we conclude that the coronary flow for WT mice during reperfusion recovered to the level at pre-ischemia.

\section{TABLE 5}

Estimates of fixed-effects for the integrated mixed-effects model (E 3.5) for coronary flow

\begin{tabular}{crrcrr}
\hline Parameter & Estimate & S.E. & $95 \%$ C.I. & Z & P-value \\
\hline $\boldsymbol{\beta}_{\mathbf{1 1}}$ & 2.86 & 0.24 & {$[2.37,3.35]$} & 11.47 & 0.00 \\
$\boldsymbol{\beta}_{\mathbf{2 1}}$ & -0.28 & 0.36 & {$[-0.99,0.44]$} & -0.75 & 0.44 \\
$\boldsymbol{\beta}_{\mathbf{1 2}}$ & 0.00 & 0.01 & {$[-0.02,0.03]$} & 0.27 & 0.79 \\
$\boldsymbol{\beta}_{\mathbf{2 2}}$ & 0.02 & 0.02 & {$[-0.02,0.05]$} & 1.02 & 0.31 \\
$\boldsymbol{\beta}_{\mathbf{1 3}}$ & 2.95 & 0.28 & {$[2.40,3.50]$} & 10.51 & 0.00 \\
$\boldsymbol{\beta}_{\mathbf{2 3}}$ & -1.04 & 0.41 & {$[-1.84,-0.23]$} & -2.52 & 0.01 \\
$\boldsymbol{\beta}_{\mathbf{1 4}}$ & 1.66 & 0.42 & {$[0.84,2.48]$} & 3.94 & 0.00 \\
$\boldsymbol{\beta}_{\mathbf{2 4}}$ & -0.23 & 0.61 & {$[-0.97,1.43]$} & 0.38 & 0.71 \\
$\boldsymbol{\beta}_{\mathbf{1 5}}$ & -1.08 & 0.12 & {$[-1.31,-0.84]$} & -8.91 & 0.00 \\
$\boldsymbol{\beta}_{\mathbf{2 5}}$ & -0.19 & 0.19 & {$[-0.56,0.18]$} & -1.00 & 0.32 \\
$\boldsymbol{\beta}_{\mathbf{1 6}}$ & -1.09 & 0.12 & {$[-1.33,-0.85]$} & -9.02 & 0.00 \\
$\boldsymbol{\beta}_{\mathbf{2 6}}$ & -0.18 & 0.19 & {$[-0.55,0.19]$} & -0.96 & 0.34 \\
$\boldsymbol{\beta}_{\mathbf{1 7}}$ & 250.54 & 44.76 & {$[163.31,3337.76]$} & 5.60 & 0.00 \\
$\boldsymbol{\beta}_{\mathbf{2 7}}$ & -32.95 & 83.24 & {$[-195.16,129.26]$} & -0.40 & 0.69 \\
\hline
\end{tabular}




\section{TABLE 6}

Estimates of variance components for the integrated mixed-effects model (E 3.5) for coronary flow

\begin{tabular}{cccccc}
\hline Parameter & Estimate & Parameter & Estimate & Parameter & Estimate \\
\hline $\boldsymbol{\sigma}_{\mathbf{1}}$ & 0.65 & $\boldsymbol{\rho}_{\mathbf{1 5}}$ & -0.30 & $\boldsymbol{\rho}_{\mathbf{3 5}}$ & 0.17 \\
$\boldsymbol{\sigma}_{\mathbf{2}}$ & 0.02 & $\boldsymbol{\rho}_{\mathbf{1 6}}$ & -0.30 & $\boldsymbol{\rho}_{\mathbf{3 6}}$ & 0.16 \\
$\boldsymbol{\sigma}_{\mathbf{3}}$ & 0.74 & $\boldsymbol{\rho}_{\mathbf{1 7}}$ & 0.07 & $\boldsymbol{\rho}_{\mathbf{3 7}}$ & 0.73 \\
$\boldsymbol{\sigma}_{\mathbf{4}}$ & 1.06 & $\boldsymbol{\sigma}_{\mathbf{2 3}}$ & -0.16 & $\boldsymbol{\sigma}_{\mathbf{4 5}}$ & -0.46 \\
$\boldsymbol{\sigma}_{\mathbf{5}}$ & 0.31 & $\boldsymbol{\rho}_{\mathbf{2 4}}$ & -0.66 & $\boldsymbol{\rho}_{\mathbf{4 6}}$ & -0.46 \\
$\boldsymbol{\sigma}_{\mathbf{6}}$ & 0.31 & $\boldsymbol{\rho}_{\mathbf{2 5}}$ & 0.66 & $\boldsymbol{\rho}_{\mathbf{4 7}}$ & 0.02 \\
$\boldsymbol{\sigma}_{\mathbf{7}}$ & 38.61 & $\boldsymbol{\rho}_{\mathbf{2 6}}$ & 0.66 & $\boldsymbol{\rho}_{\mathbf{5 6}}$ & 1.00 \\
$\boldsymbol{\rho}_{\mathbf{1 2}}$ & -0.08 & $\boldsymbol{\rho}_{\mathbf{2 7}}$ & 0.24 & $\boldsymbol{\rho}_{\mathbf{5 7}}$ & 0.71 \\
$\boldsymbol{\rho}_{\mathbf{1 3}}$ & 0.57 & $\boldsymbol{\rho}_{\mathbf{3 4}}$ & 0.63 & $\boldsymbol{\rho}_{\mathbf{6 7}}$ & 0.71 \\
$\boldsymbol{\rho}_{\mathbf{1 4}}$ & 0.44 & & & & \\
\hline
\end{tabular}

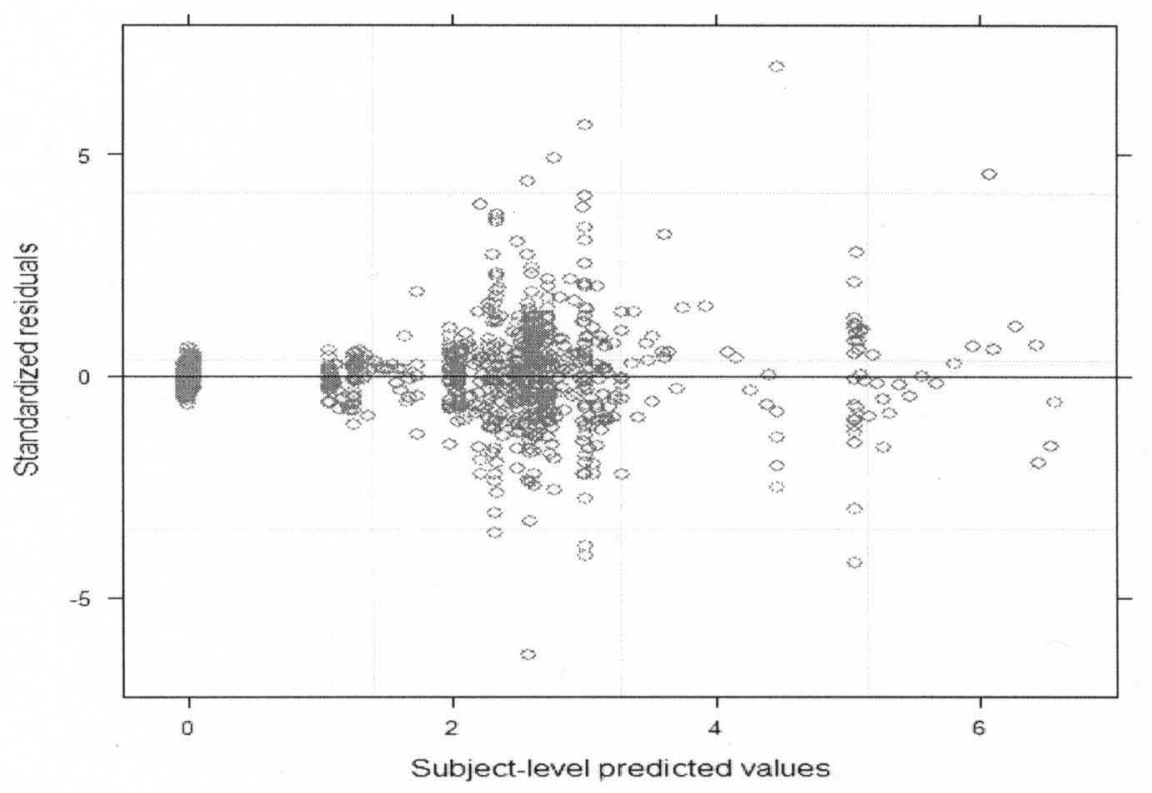

Figure 9. Diagnostic plot: Standardized residuals versus subiect-level fitted 


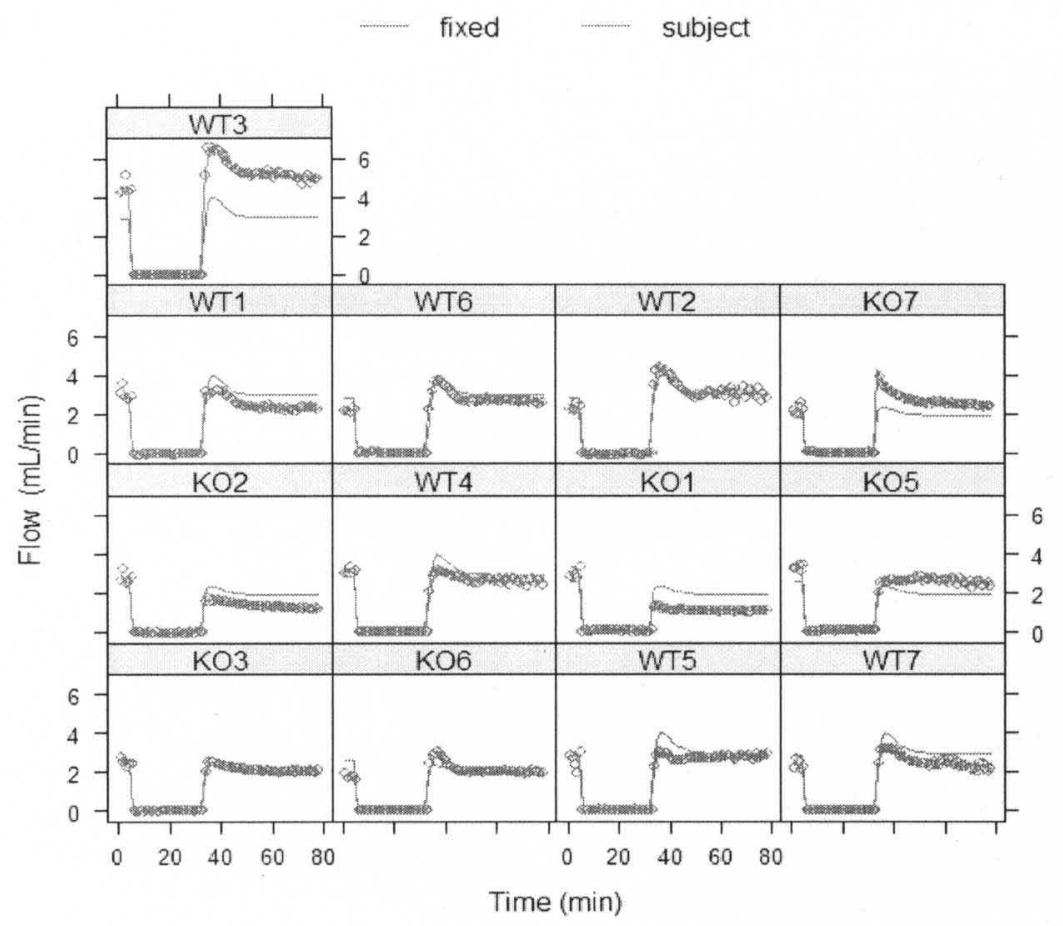

Figure 10. Plots of the fitted coronary flows at group-level and the predicted coronary flows at subject-level, as well as the observed coronary flows (circles) versus time for the entire experimental data.

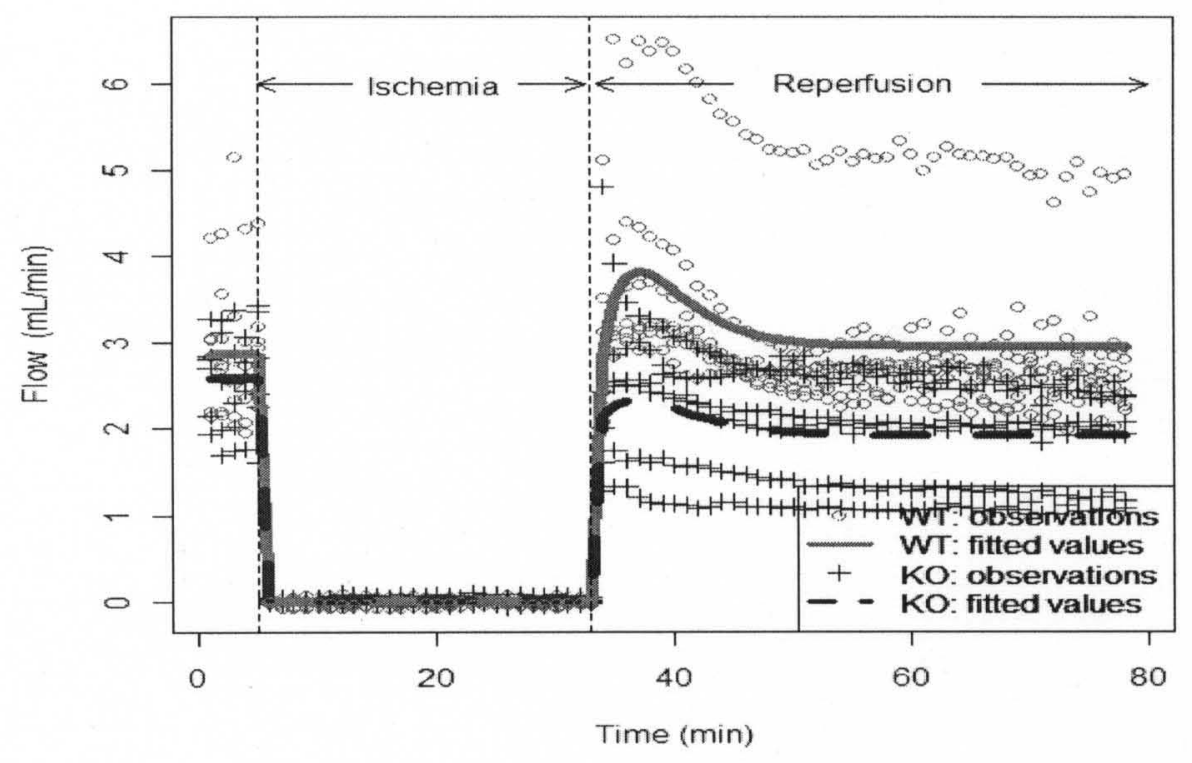

Figure 11. Fitted and observed coronary flows versus time. 
To test whether the coronary flow during reperfusion recovers to the level at preischemia for $\mathrm{KO}$ mice, the underlying null hypothesis is $H_{0}: \beta_{11}+\beta_{21}-\beta_{13}-\beta_{23}=0$ and the alternative hypothesis is $H_{1}: \beta_{11}+\beta_{21}-\beta_{13}-\beta_{23} \neq 0$.

The Wald test statistic is:

$$
z=\frac{\lambda^{\prime} \hat{\beta}}{\sqrt{\lambda^{\prime} \widehat{\operatorname{Cov}}(\hat{\beta}) \lambda}}=2.526
$$

where $\lambda^{\prime}=(1,1,0,0,0,0,-1,-1,0,0,0,0,0,0)$ and $P$-value is $2 P(\mathrm{z} \geq 2.526)=0.012$.

Because $P$-value is 0.012 , we reject $H_{0}$ at the $5 \%$ significance level. Therefore, we conclude that the coronary flow for KO mice after reperfusion does not recover to the level of pre-ischemia.

Based on the two hypothesis tests, we conclude that the recovery rate of the coronary flow is better in WT mice than in KO mice.

\subsection{Left ventricle (LV) developed pressure}

Figure 3 illustrates the LV developed pressure over different period of time. Particularly, the LV developed pressure during reperfusion was constant and increased exponentially. The onset point to exponential growth is called a change point. The onset of LV developed pressure is important since it indicates how rapidly the LV developed pressure starts to recover. The asymptotic value for the LV developed pressure is also important since it indicates the degree of recovery. We propose the following model: 


$$
y(t)= \begin{cases}C_{1} & t \leq C_{2} \\ C_{1}+C_{3}\left(1-e^{-C_{4}\left(t-C_{2}\right)}\right) & t \geq C_{2}\end{cases}
$$

to describe the $\mathrm{LV}$ developed pressure during reperfusion, where $C_{2}$ is the change point, and $C_{1}+C_{3}$ is the asymptotic LV developed pressure. Note that the true time of transition from the constant to the exponential growth is unknown for each mouse and is influenced by random factors, so the change point was estimated by including a random effect in the model [20]. We propose the following nonlinear mixed-effects change point model:

$$
y_{i j}=\left\{\begin{array}{cc}
C_{1 i} & t_{i j} \leq C_{2 i} \\
C_{1 i}+C_{3 i}\left[1-\exp \left(-C_{4 i}\left(t_{i j}-C_{2 i}\right)\right)\right] & t_{i j} \geq C_{2 i},
\end{array}\right.
$$

where

$$
\begin{gathered}
C_{k i}=\beta_{1 k}+\beta_{2 k} \text { type }_{i}+b_{k i}, \quad k=1, \ldots, 4, \\
\text { type }_{i}=\left\{\begin{array}{cc}
1 & \text { if mouse } i \text { is knockout } \\
0 & \text { if mouse } i \text { is wild - type } .
\end{array}\right.
\end{gathered}
$$

$C_{1 i}$ is constant $\mathrm{LV}$ developed pressure before the onset of exponential growth, $C_{2 i}$ represents the unknown transition time between the constant LV developed pressure and the exponential growth, $C_{1 i}+C_{3 i}$ is the asymptotic LV developed pressure as $t_{i j} \rightarrow \infty$, and $C_{4 i}$ is the exponential constant growth rate for the exponential LV developed pressure. Table 7 shows the estimated parameters and Figure 12 shows the fitted results for LV developed pressure during reperfusion. LV developed pressure recovered to a much greater extent in WT hearts than in $\mathrm{KO}$ hearts and there was no significant difference in transition time between the two types of mice. An overall assessment of the nonlinear mixed-effects change point model (E 3.6) is shown in Figure 13. Both the fitted LV developed pressures at group-level and the predicted LV developed pressures at 
subject-level during reperfusion are displayed to show how individual effects are accounted for the nonlinear mixed-effects change point model (E 3.6). As can be seen Figure 13, the subject-level predictions matched the observed LV developed pressures very well during reperfusion, indicating the goodness of fit of the model.

$\underline{\text { Statistical test and inference for LV developed pressure }}$

$\hat{\beta}_{12}=49.67$ with $p$-value $<0.01$ indicates that the transition time is about $49.67-33$

$=16.67$ minutes from starting reperfusion. To test whether there is a significant

difference between WT mice and KO mice in time at the onset of the recovery in LV

\section{TABLE 7}

Estimates of fixed-effects and variance components for nonlinear mixed-effects change point model (E3.6) for left ventricle (LV) developed pressure during reperfusion

\begin{tabular}{crrcrr}
\hline Parameter & Estimate & S.E. & $95 \%$ C.I. & $Z$ & P-value \\
\hline $\boldsymbol{\beta}_{\mathbf{1 1}}$ & 3.22 & 0.35 & {$[2.54,3.90]$} & 9.24 & 0.00 \\
$\boldsymbol{\beta}_{\mathbf{2 1}}$ & -0.17 & 0.51 & {$[-1.16,0.83]$} & -0.33 & 0.74 \\
$\boldsymbol{\beta}_{\mathbf{1 2}}$ & 49.67 & 1.24 & {$[47.26,52.09]$} & 40.15 & 0.00 \\
$\boldsymbol{\beta}_{\mathbf{2 2}}$ & 0.55 & 1.90 & {$[-3.15,4.25]$} & 0.29 & 0.77 \\
$\boldsymbol{\beta}_{\mathbf{1 3}}$ & 25.10 & 3.40 & {$[18.47,31.73]$} & 7.39 & 0.00 \\
$\boldsymbol{\beta}_{\mathbf{2 3}}$ & -10.48 & 5.01 & {$[-20.26,-0.70]$} & -2.09 & 0.03 \\
$\boldsymbol{\beta}_{\mathbf{1 4}}$ & -2.36 & 0.10 & {$[-2.56,-2.16]$} & -22.80 & 0.00 \\
$\boldsymbol{\beta}_{\mathbf{2 4}}$ & -0.31 & 0.19 & {$[-0.68,0.05]$} & -1.67 & 0.10 \\
$\boldsymbol{\sigma}_{\mathbf{1}}$ & 0.79 & & {$[0.48,1.32]$} & & \\
$\boldsymbol{\sigma}_{\mathbf{2}}$ & 3.18 & & {$[2.05,4.95]$} & & \\
$\boldsymbol{\sigma}_{\mathbf{3}}$ & 8.76 & & {$[5.90,13.01]$} & & \\
$\boldsymbol{\sigma}_{\mathbf{4}}$ & 0.20 & & {$[0.06,0.65]$} & & \\
$\boldsymbol{\rho}_{\mathbf{1 2}}$ & -0.60 & & {$[-0.91,0.16]$} & & \\
$\boldsymbol{\rho}_{\mathbf{1 3}}$ & 0.17 & & {$[-0.44,0.68]$} & & \\
$\boldsymbol{\rho}_{\mathbf{1 4}}$ & 0.49 & & {$[-0.54,0.93]$} & & \\
$\boldsymbol{\rho}_{\mathbf{2 3}}$ & -0.73 & & {$[-0.93,-0.22]$} & & \\
$\boldsymbol{\rho}_{\mathbf{2 4}}$ & -0.55 & & {$[-0.91,0.30]$} & & \\
$\boldsymbol{\rho}_{\mathbf{3 4}}$ & 0.04 & & {$[-0.51,0.58]$} & & \\
\hline
\end{tabular}




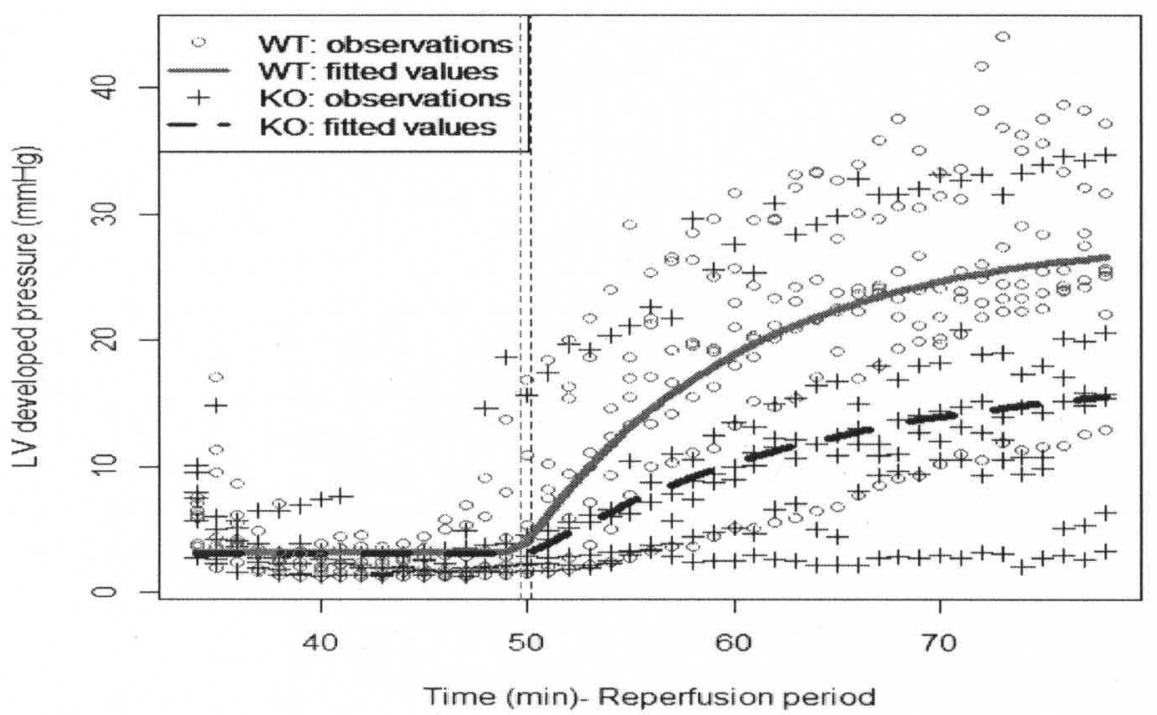

Figure 12. Fitted and observed LV developed pressures versus time during reperfusion.

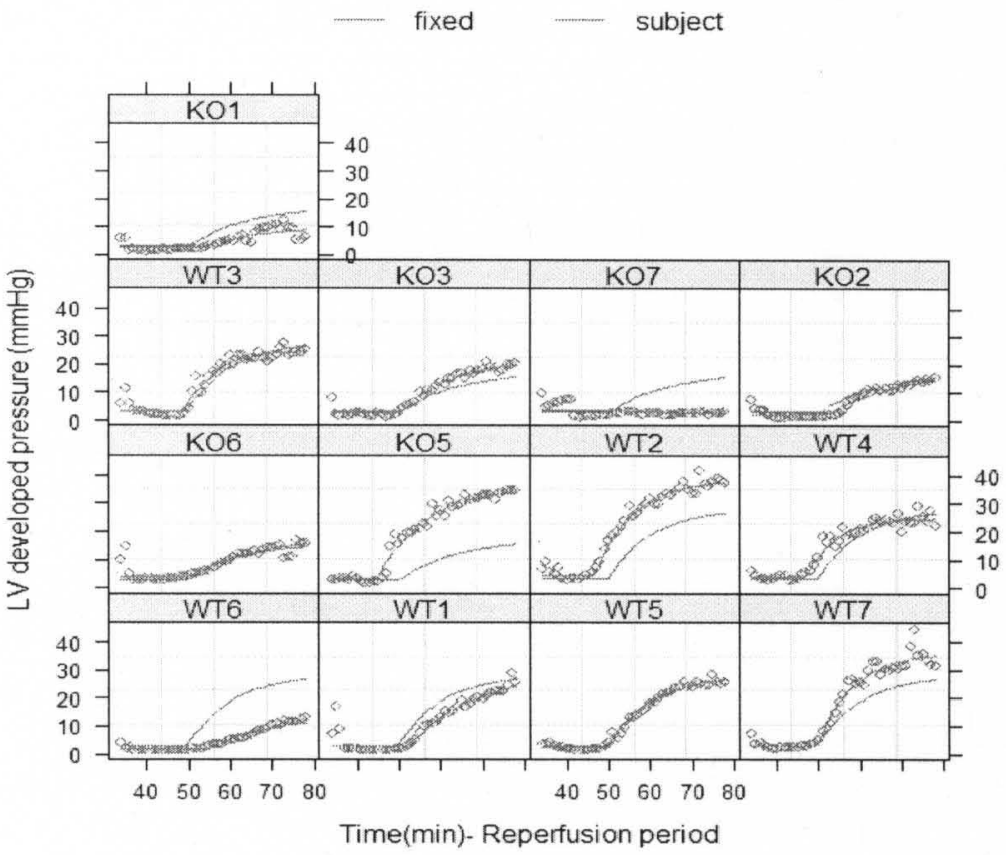

Figure 13. Plots of the fitted LV developed pressures at group-level and the predicted LV developed pressures at subject-level, as well as observed LV developed pressures (circles) versus time during reperfusion. 
developed pressure during reperfusion, the underlying null hypothesis is $H_{0}: \beta_{22}=0$ and the alternative hypothesis is $H_{1}: \beta_{22} \neq 0$.

The Wald test statistic is:

$$
z=\frac{\hat{\beta}_{22}}{\sqrt{\operatorname{Var}\left(\hat{\beta}_{22}\right)}}=0.29
$$

$P$-value is $2 P(z \geq 0.29)=0.77$, which indicates that we cannot reject $H_{0}$ at the $5 \%$ significance level. Therefore, we conclude that there is no significant difference in time at the onset of the recovery in LV developed pressure during reperfusion between KO-type and WT mice.

To test whether the LV developed pressures between WT mice and KO mice after reperfusion are significantly different, the null hypothesis is $H_{0}: \beta_{23}=0$, and the alternative is $H_{1}: \beta_{23} \neq 0$.

The Wald test statistic is:

$$
z=\frac{\hat{\beta}_{23}}{\sqrt{\operatorname{Var}\left(\hat{\beta}_{23}\right)}}=-2.09,
$$

$P$-value $=2 P(z \geq|-2.09|)=0.03$, which indicates that we reject $H_{0}$ at the $5 \%$ significance level. The results show that the asymptotic LV developed pressure during reperfusion is significantly higher in WT mice than that in KO mice. 


\section{CHAPTER 4}

\section{Discussion and conclusions}

The defining feature of a longitudinal study is that subjects are measured repeatedly over time. As repeated observations made on the same subject tend to be correlated, longitudinal data require specific statistical methods to account for the correlation. Mixed-effects model is an efficient tool for analyzing longitudinal data. It provides covariance structure for correlated repeated measurements. Unlike traditional repeated measures ANOVA, the time points are not fixed and all available data can be used in mixed-effects model, provided data are missing at random.

In this study, we focus on applying the mixed-effects models to the repeated measurements of cardiac function including heart rate, coronary flow, and left ventricle developed pressure in the isolated, Langendorff perfused hearts of the Gluatathione-Stransferase (GSTP) gene KO and WT mice following ischemia/ reperfusion injury. Each aspect of the cardiac function data consists of three time periods: pre-ischemia, ischemia, and reperfusion periods. We developed piecewise nonlinear mixed-effects model to describe the different aspects of cardiac function. We examined how cardiac function was altered by ischemia/reperfusion injury for KO mice and WT mice using nonlinear mixed-effects model and change point model. The Wald test and Akaike Information criterion (AIC) 
are applied to obtain a parsimonious model for each aspect of cardiac function. The goodness of fit is examined, and the proposed models fit the experimental data very well. Nonlinear mixed-effects models provide not only interpretable models for the experimental data but also reliable predictions for the response variables in our experimental data. Therefore, nonlinear mixed-effects models are highly recommended for the assessment of the dynamic change of the cardiac function and the assessment of the treatment effect on the cardiac function. 


\section{REFERENCES}

[1] J. Twisk, Applied longitudinal data analysis for epidemiology: a practical guide, Cambridge University Press, New York, 2003.

[2] R. D. Wimmer and J. R. Dominick, Mass Media Research: An Introduction, Thomson Wadsworth, Belmont, 2005.

[3] D. Hedeker and R. D. Gibbons, Longitudinal data analysis, John Wildey and Sons, New Jersey, 2006.

[4] G. Fitzmaurice, M. Davidian M, G. Verbeke, and G. Molenberghs, Longitudinal Data Analysis: A Handbook of Modern Statistical Methods, Chapman \& Hall/CRC, 2008.

[5] C. Krueger and L. Tian, "A comparison of the general linear mixed model and repeated measures ANOVA using a dataset with multiple missing data points," Biological Research Nursing, Vol. 6, pp. 151-157, 2004.

[6] N. M. Laird and J. H. Ware, "Random-effects models for longitudinal data," Biometrics, vol. 38, pp. 963-974, 1982.

[7] A. P. Dempster, D. B. Rubin, and R. K. Tsutakawa, "Estimation in covariance component models," Journal of the American Statistical Society, vol. 76, pp.341-353, 1981.

[8] H. Goldstein, Multilevel Statistical Models, $2^{\text {nd }}$ edition, Halstead Press, New York, 1995.

[9] S. W. Raudenbush and A. S. Bryk, Hierarchical Linear Models, $2^{\text {nd }}$ edition, Sage, Thousand Oaks, CA, 2002.

[10] R. D. Bock, Measurement of human variation: a two stage model, in Multilevel Analysis of Educational Data, ed. R.D. Bock, Academic Press, New York, pp. 319$342,1989$.

[11] J. D. Leeuw and I. Kreft, "Random coefficient models for multilevel analysis," Journal of Educational Statistics, vol. 11, pp. 57-85, 1986.

[12] J. S. Long, Regression Models for Categorical and Limited Dependent Variables, Sage Publications, Thousand Oaks, CA, 1997. 
[13] S. L. Hui and J. O. Berger, "Empirical Bayes estimation of rates in longitudinal studies," Journal of American Statistical Association, vol. 78, pp. 753-759, 1983.

[14] R. D. Bock, "Within-subject experimentation in psychiatric research," In R. D. Gibbons and M. W. Dysken, editiors, Statistical and Methodological Advances in Psychiatric Research, pp. 59-90, Spectrum, New York, 1983b.

[15] M. J. Lindstrom and D. M. Bates, "Nonlinear mixed effects models for repeated measures data," Biometrics, vol. 46, pp.673-687, 1990.

[16] R. Cudeck and J. R. Harring, "Analysis of nonlinear patterns of change with random coefficient models," Annual Review of Psychology, vol. 58, pp. 615-637, 2007.

[17] J. C. Pinheiro and M. B. Douglas, Mixed-Effects Models in S and S-PLUS, Springer, New York, 2000.

[18] G. M. Fitzmaurice, N. M. Laird, and J. H. Ware, Applied Longitudinal Analysis, John Wiley, New York 2004.

[19] B. T. West, K. B. Welch, and A. T. Andrzej, Linear Mixed Models: A practical Guide Using Statistical Software, Chapman \& Hall/CRC, 2007.

[20] H. Akaike, "Information theory and an extension of the maximum likelihood principle," 2nd International Symposium on Information Theory, ed. B.N. Petrov and F. Csaki, pp. 267-281, Akademiai Kiado, Budapest, 1973.

[21] G. Schwarz, "Estimating the dimension of a model," Annlas of statistics, vol. 6, pp. 461-464, 1978.

[22] U. Schulze, "A method of Estimation of Change Points in Multiphasic Growth Models," Biometrical Journal, vol. 26, pp. 495-504, 1984.

[23] K. D. Tew and D. M. Townsend, "Regulatory functions of glutathione S-transferase P1-1 unrelated to detoxification," drug metabolism reviews, pp. 1-15, 2011.

[24] D. J. Conklin, P. Habetzettl, and A. Bhatnagar, "Glutathione-S-transferase P protects against endothelial dysfunction induced by tobacco smoke," American Journal of Physiology: Heart and Circulatory Physiology, vol. 296, pp. H1586-1597, 2009.

[25] D. J. Conklin, B. G. Hill, K. Kaiserova, Y. Guo, R. Bolli, and A. Bhatnagar, "Deficiency of Glutathione-S-transferase P (GSTP)-mediated Detoxification of Lipid Peroxidation Products Increases Myocardial Ischemia-Reperfusion Injury," Circulation, vol. 118, pp. S546 - S547, 2008. 


\section{Appendix A: Linear mixed-effects model for single period}

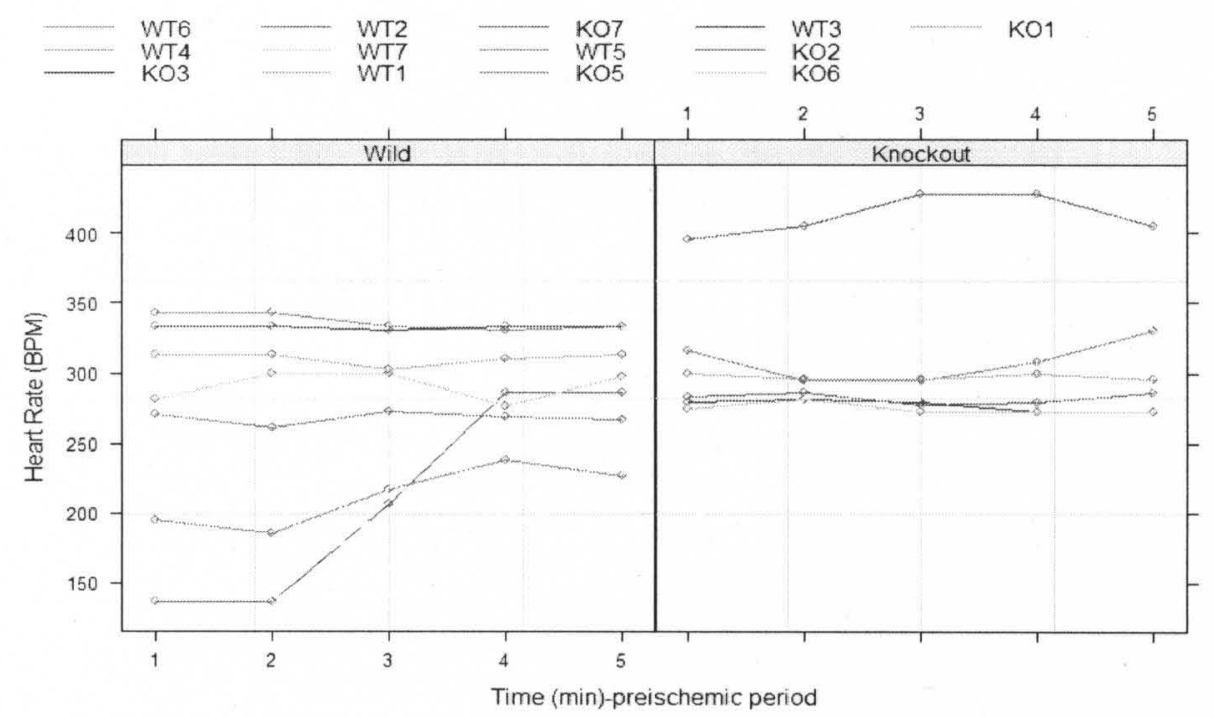

Figure 14. Heart rates versus time during pre-ischemia.

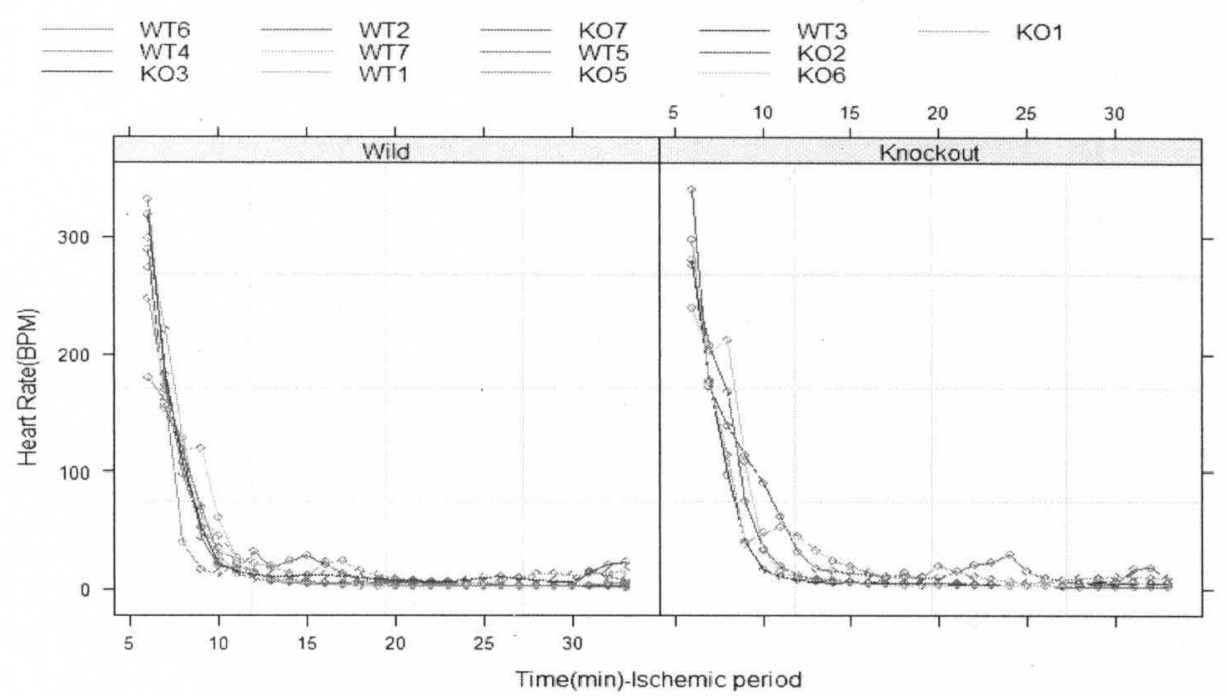

Figure 15. Heart rates versus time during ischemia. 


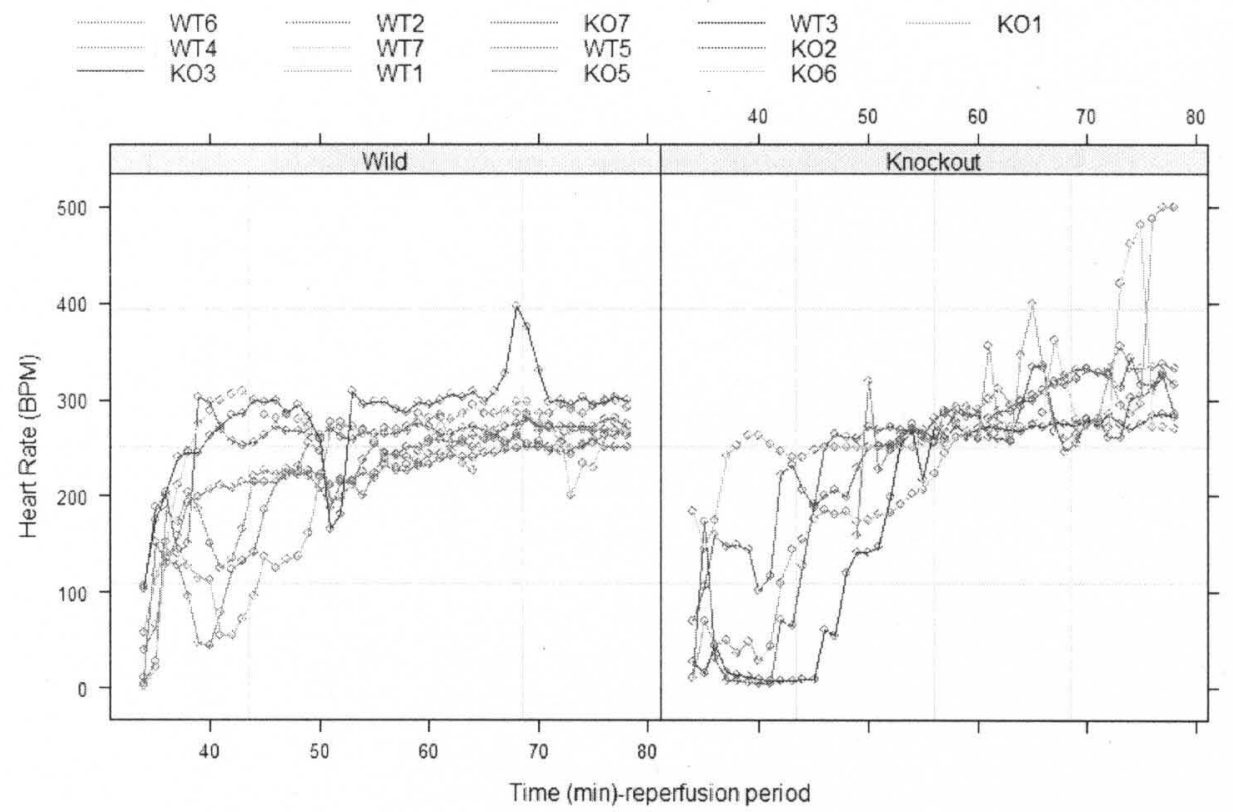

Figure 16. Heart rates versus time during reperfusion.

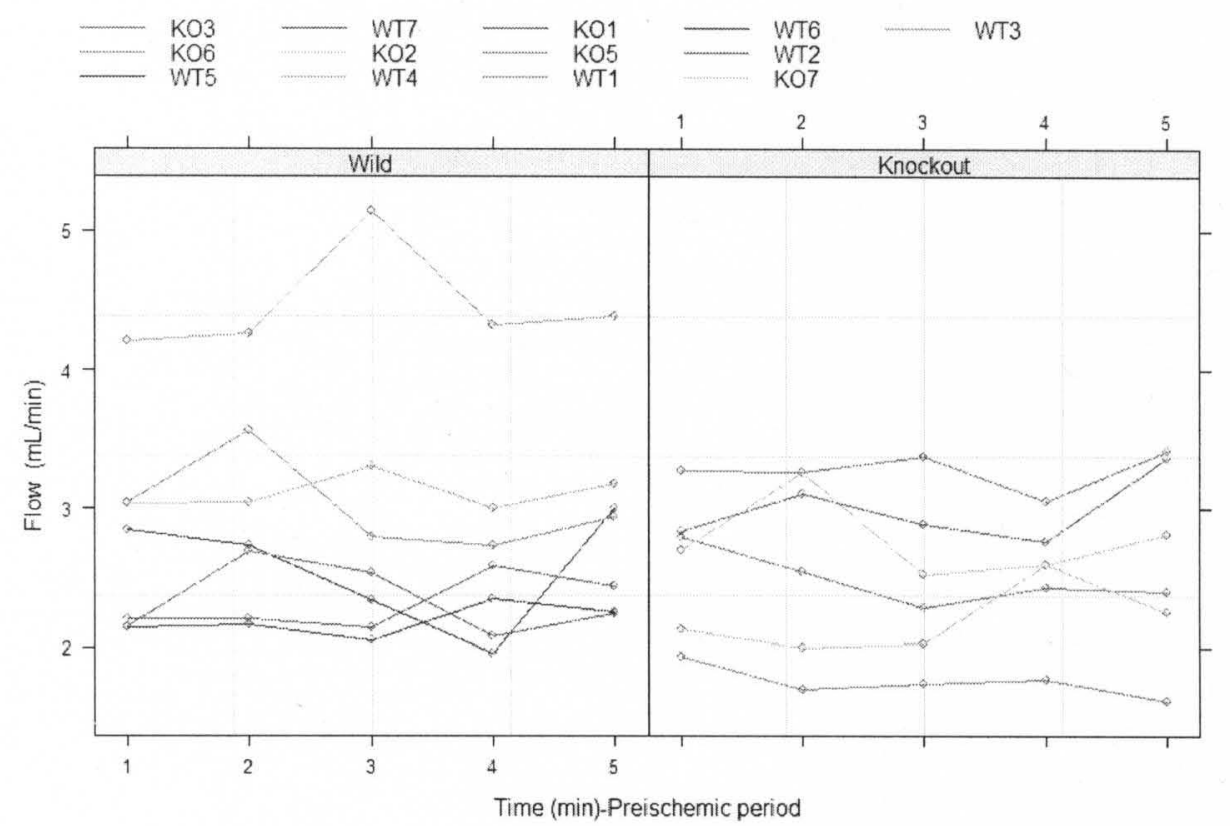

Figure 17. Coronary flows versus time during pre-ischemia. 


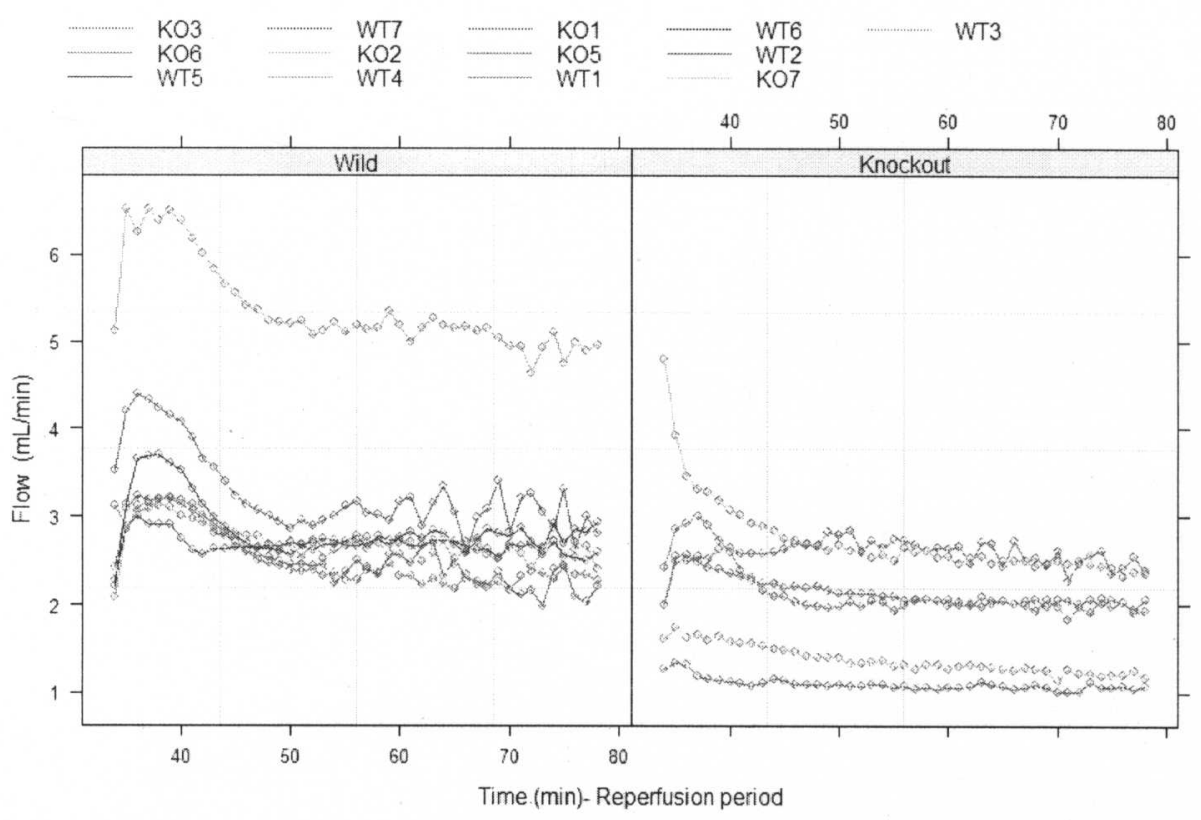

Figure 18. Coronary flows versus time during reperfusion.

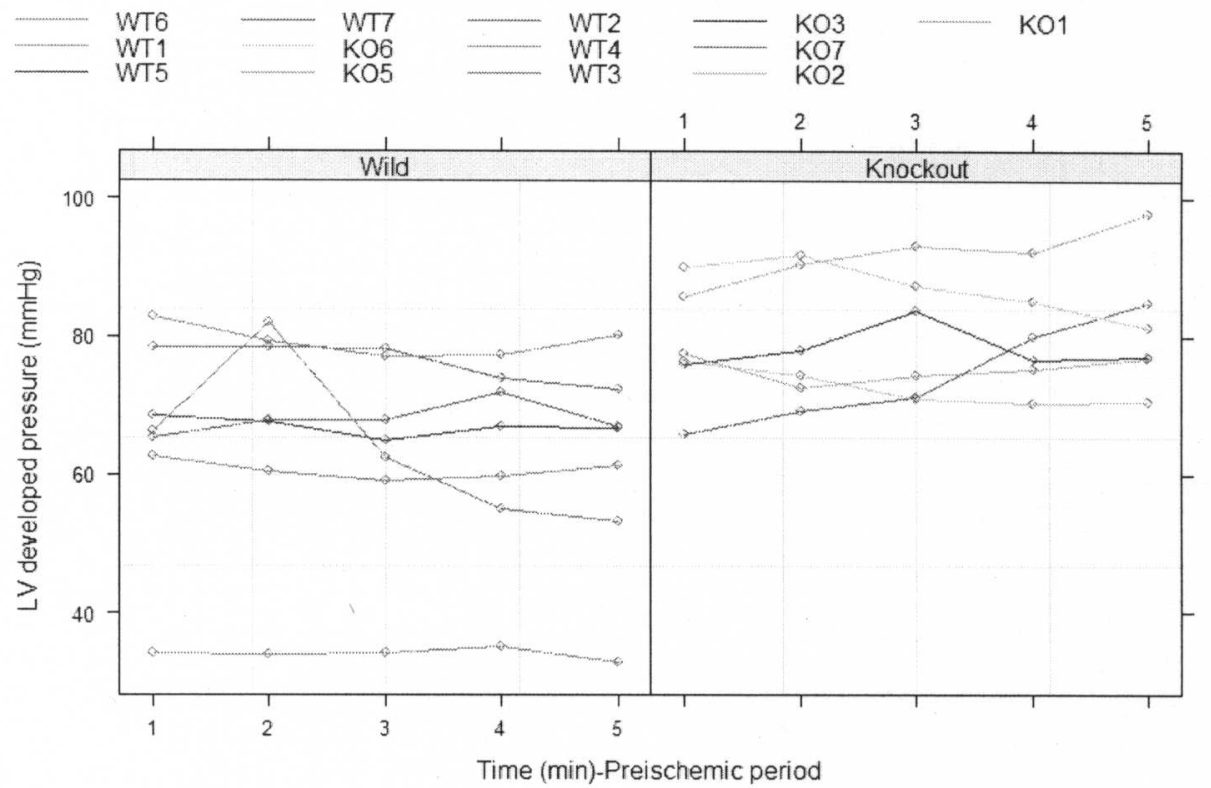

Figure 19. Left ventricle (LV) developed pressures versus time during preischemia. 


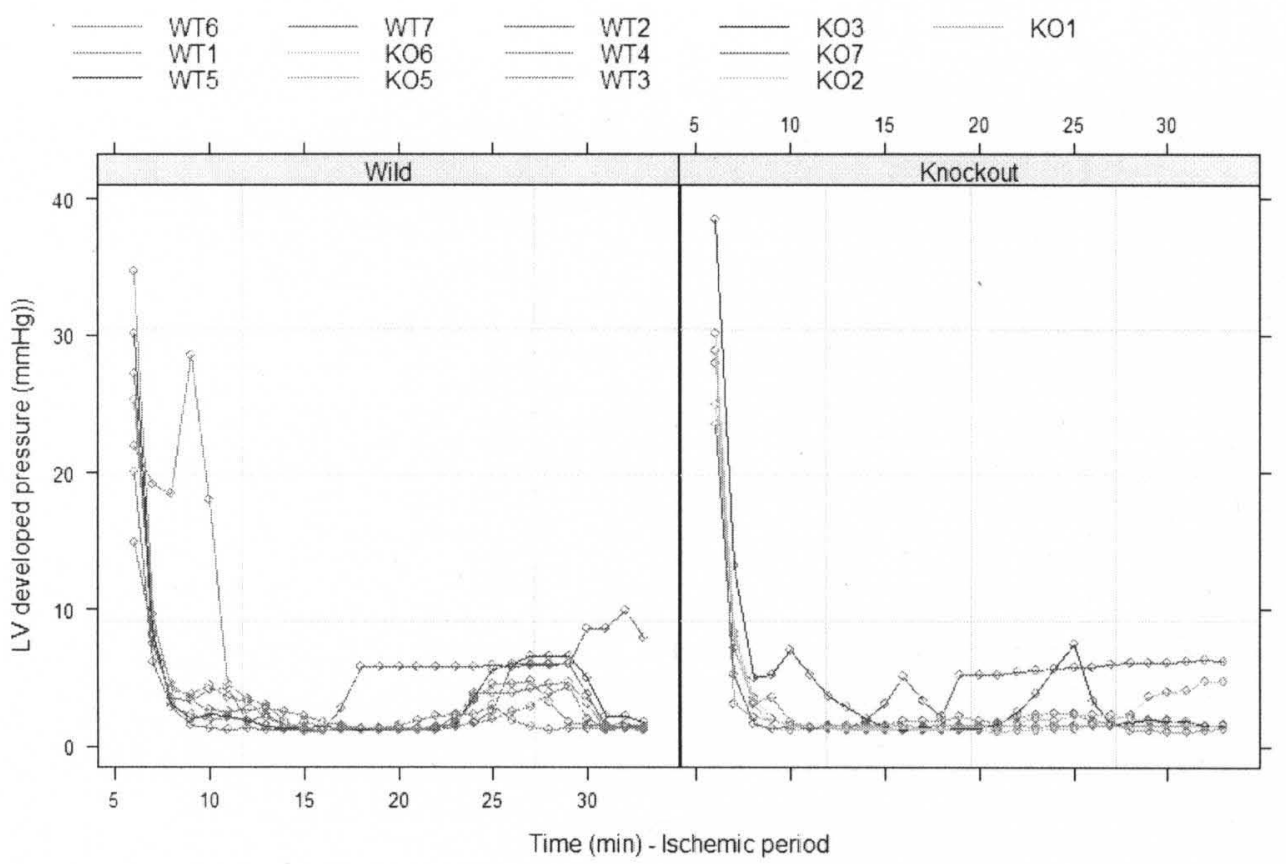

Figure 20. LV developed pressures versus time during ischemia.

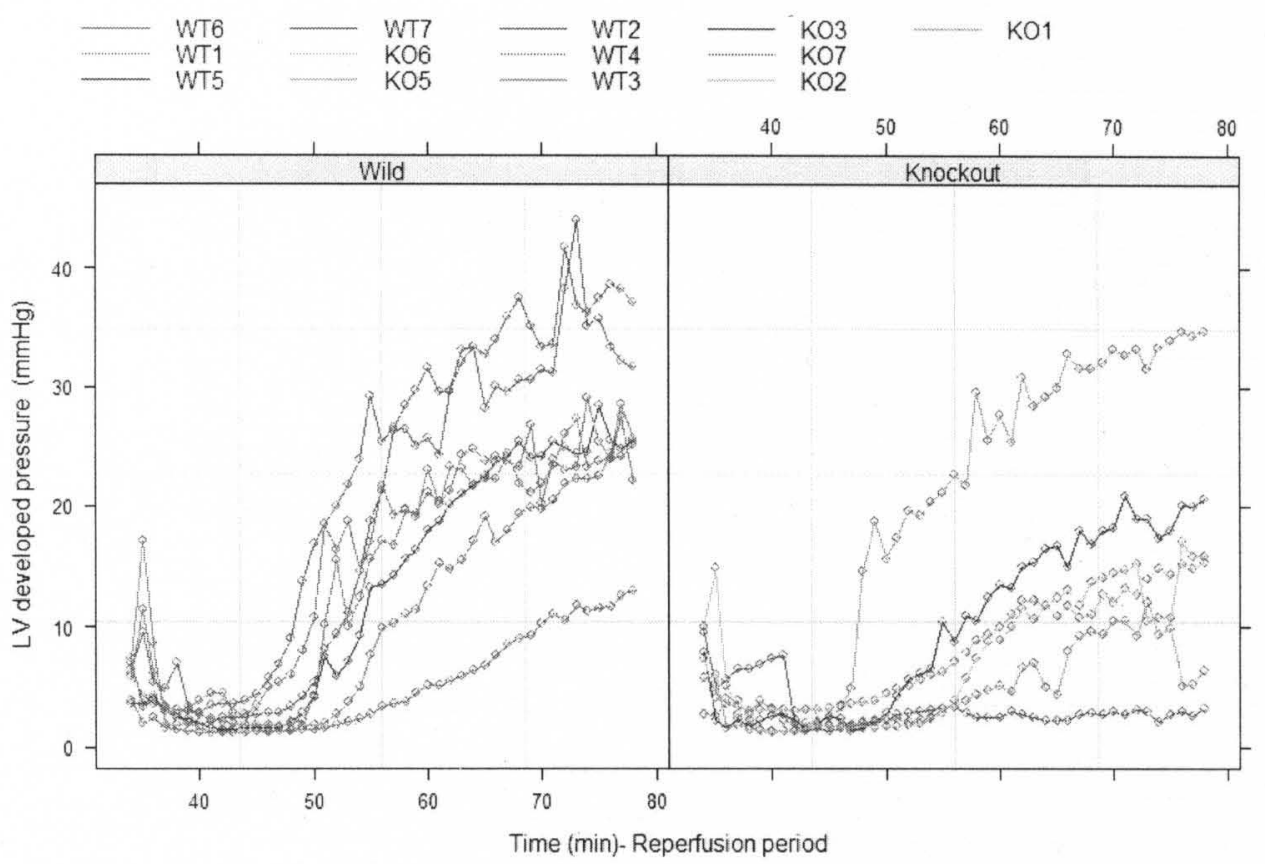

Figure 21. LV developed pressures versus time during reperfusion. 
TABLE 8

Estimates of fixed-effects for linear mixed-effects model (E 3.1) for heart rate during preischemia

\begin{tabular}{crrrrr}
\hline Parameter & Estimate & S.E. & DF & Z & P-value \\
\hline $\boldsymbol{\beta}_{\mathbf{0}}$ & 257.12 & 29.66 & 50 & 8.67 & 0.00 \\
$\boldsymbol{\beta}_{\mathbf{1}}$ & 7.71 & 4.81 & 50 & 1.60 & 0.12 \\
$\boldsymbol{\beta}_{\mathbf{2}}$ & 49.59 & 43.66 & 11 & 1.14 & 0.28 \\
$\boldsymbol{\beta}_{\mathbf{3}}$ & -6.98 & 7.08 & 50 & -0.99 & 0.33 \\
\hline
\end{tabular}

TABLE 9

Estimates of fixed-effects for linear mixed-effects model (E 3.1) for heart rate during ischemia

\begin{tabular}{crrrrr}
\hline Parameter & Estimate & S.E. & DF & Z & P-value \\
\hline $\boldsymbol{\beta}_{\mathbf{0}}$ & 114.58 & 9.56 & 322 & 11.99 & 0.00 \\
$\boldsymbol{\beta}_{\mathbf{1}}$ & -4.38 & 0.45 & 322 & -9.67 & 0.00 \\
$\boldsymbol{\beta}_{\mathbf{2}}$ & 17.00 & 14.81 & 10 & 1.15 & 0.28 \\
$\boldsymbol{\beta}_{\mathbf{3}}$ & -0.62 & 0.70 & 322 & -0.89 & 0.37 \\
\hline
\end{tabular}

TABLE 10

Estimates of fixed-effects for linear mixed-effects model (E 3.1) for heart rate during reperfusion

\begin{tabular}{crrrrr}
\hline Parameter & Estimate & S.E. & DF & Z & P-value \\
\hline $\boldsymbol{\beta}_{\mathbf{0}}$ & 167.62 & 25.85 & 526 & 6.48 & 0.00 \\
$\boldsymbol{\beta}_{\mathbf{1}}$ & 3.02 & 0.71 & 526 & 4.28 & 0.00 \\
$\boldsymbol{\beta}_{\mathbf{2}}$ & -65.31 & 40.06 & 10 & -1.99 & 0.07 \\
$\boldsymbol{\beta}_{\mathbf{3}}$ & 2.76 & 1.09 & 526 & 2.85 & 0.00 \\
\hline
\end{tabular}

\section{TABLE 11}

Estimates of fixed-effects for linear mixed-effects model (E 3.1) for coronary flow during pre-ischemia

\begin{tabular}{crrrrr}
\hline Parameter & Estimate & S.E. & DF & Z & P-value \\
\hline $\boldsymbol{\beta}_{\mathbf{0}}$ & 2.86 & 0.27 & 50 & 10.27 & 0.00 \\
$\boldsymbol{\beta}_{\mathbf{1}}$ & 0.00 & 0.03 & 50 & 0.01 & 0.99 \\
$\boldsymbol{\beta}_{\mathbf{2}}$ & -0.26 & 0.41 & 11 & -0.64 & 0.54 \\
$\boldsymbol{\beta}_{\mathbf{3}}$ & -0.00 & 0.05 & 50 & -0.10 & 0.92 \\
\hline
\end{tabular}


TABLE 12

Estimates of fixed-effects for linear mixed-effects model (E 3.1) for coronary flow during reperfusion

\begin{tabular}{ccccrr}
\hline Parameter & Estimate & S.E. & DF & \multicolumn{1}{l}{ P-value } \\
\hline $\boldsymbol{\beta}_{\mathbf{0}}$ & 4.07 & 0.48 & 570 & 8.52 & 0.00 \\
$\boldsymbol{\beta}_{\mathbf{1}}$ & -0.02 & 0.03 & 570 & -4.42 & 0.00 \\
$\boldsymbol{\beta}_{\mathbf{2}}$ & -1.43 & 0.70 & 11 & -2.04 & 0.07 \\
$\boldsymbol{\beta}_{\mathbf{3}}$ & 0.01 & 0.01 & 570 & 0.99 & 0.32 \\
\hline
\end{tabular}

TABLE 13

Estimates of fixed-effects for linear mixed-effects model (E 3.1) for LV developed pressure during pre-ischemia

\begin{tabular}{crrrrr}
\hline Parameter & Estimate & S.E. & DF & Z & P-value \\
\hline $\boldsymbol{\beta}_{\mathbf{0}}$ & 67.52 & 5.47 & 50 & 12.33 & 0.00 \\
$\boldsymbol{\beta}_{\mathbf{1}}$ & -1.13 & 0.88 & 50 & -1.29 & 0.20 \\
$\boldsymbol{\beta}_{\mathbf{2}}$ & 10.48 & 8.06 & 11 & 1.30 & 0.22 \\
$\boldsymbol{\beta}_{\mathbf{3}}$ & 1.76 & 1.30 & 50 & 1.36 & 0.18 \\
\hline
\end{tabular}

TABLE 14

Estimates of fixed-effects for linear mixed-effects model (E 3.1) for LV developed pressure during reperfusion

\begin{tabular}{crrrrr}
\hline Parameter & Estimate & S.E. & DF & $Z$ & P-value \\
\hline $\boldsymbol{\beta}_{\mathbf{0}}$ & -24.01 & 4.07 & 570 & -5.90 & 0.00 \\
$\boldsymbol{\beta}_{\mathbf{1}}$ & 0.68 & 0.11 & 570 & 6.32 & 0.00 \\
$\boldsymbol{\beta}_{\mathbf{2}}$ & 12.70 & 5.99 & 11 & 2.12 & 0.06 \\
$\boldsymbol{\beta}_{\mathbf{3}}$ & -0.32 & 0.16 & 570 & -2.02 & 0.04 \\
\hline
\end{tabular}




\section{Appendix B: $\mathrm{R}$ code for the thesis}

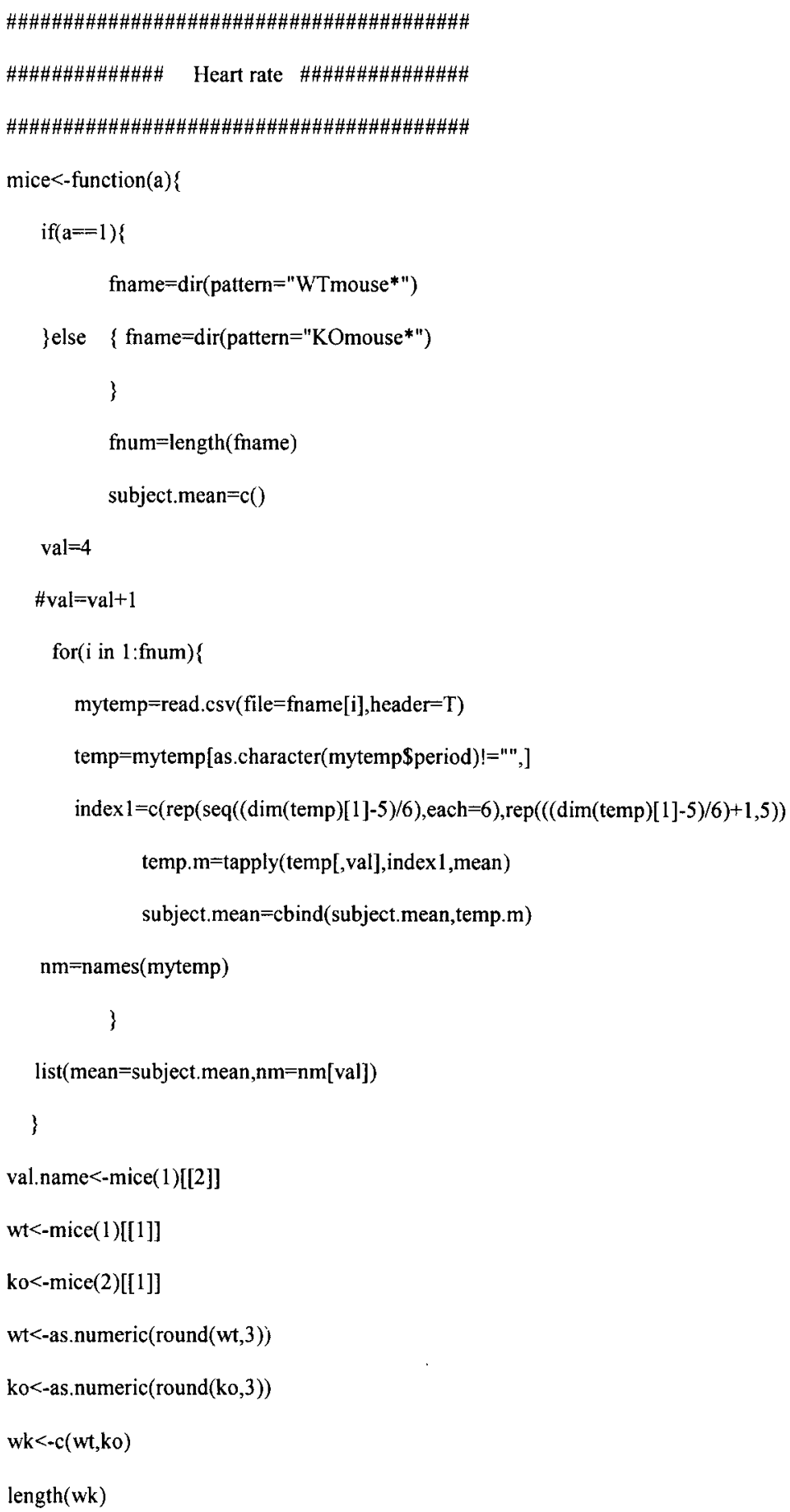




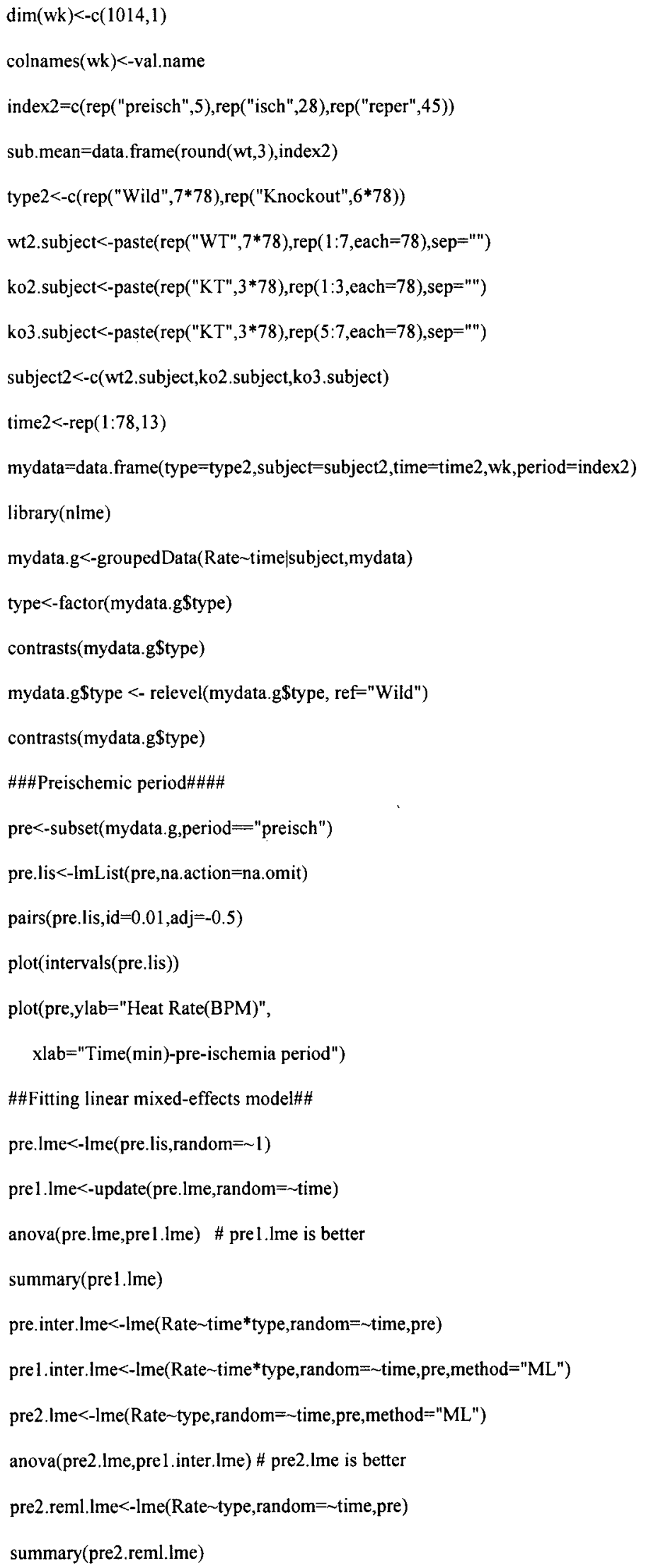




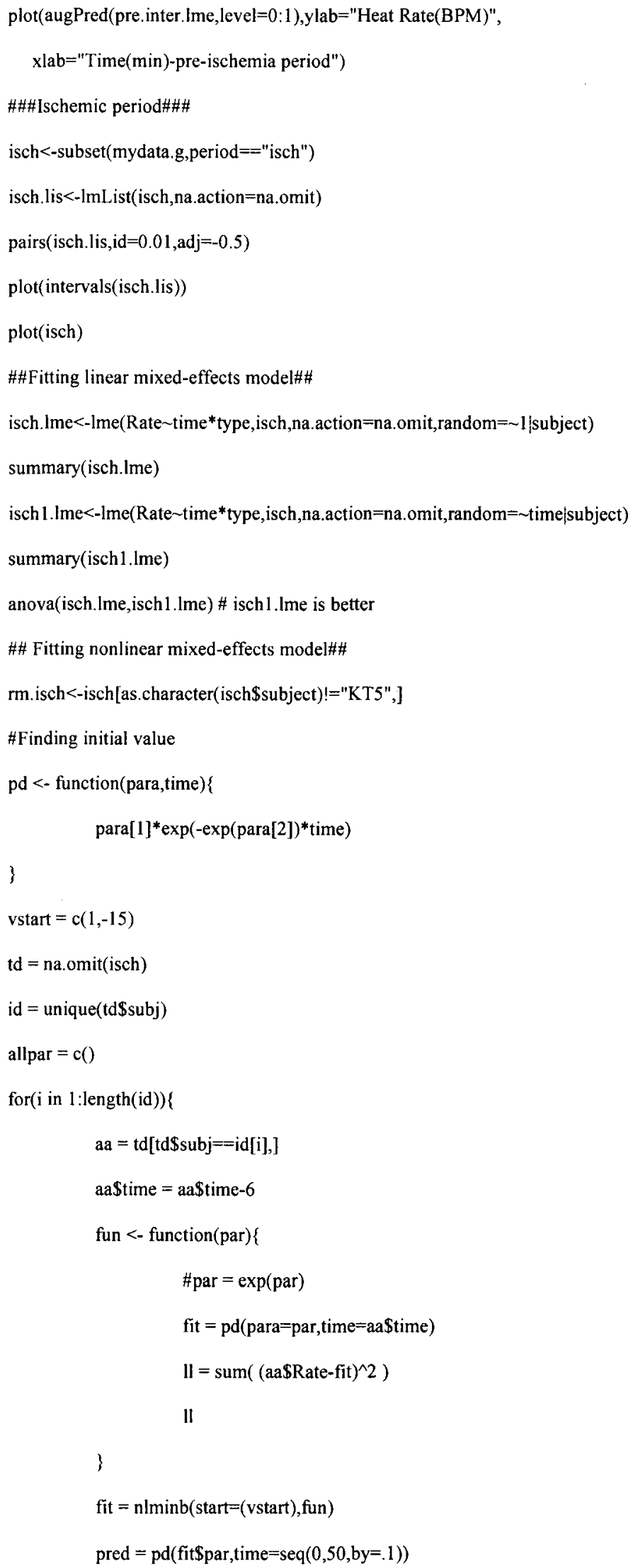




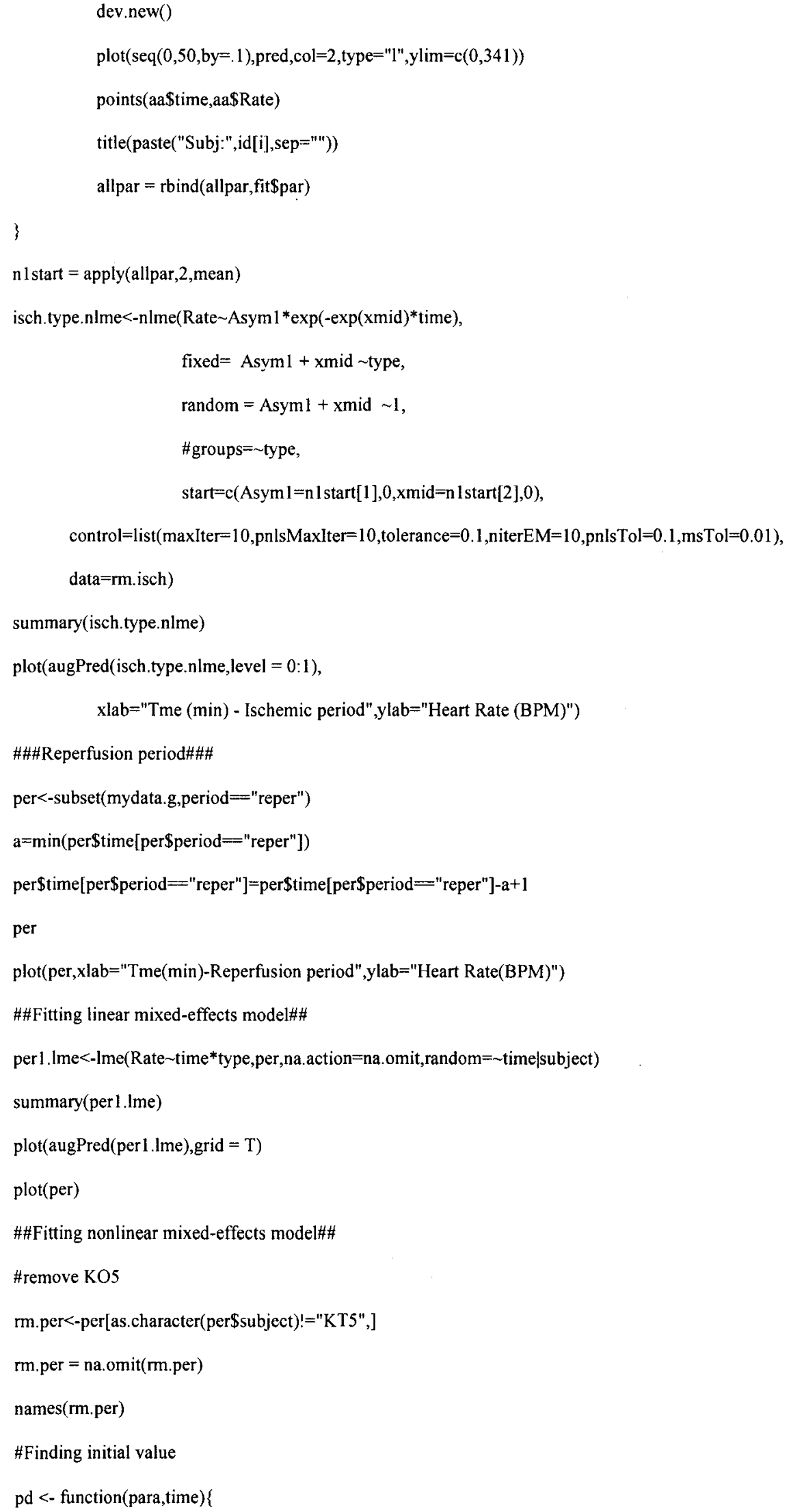




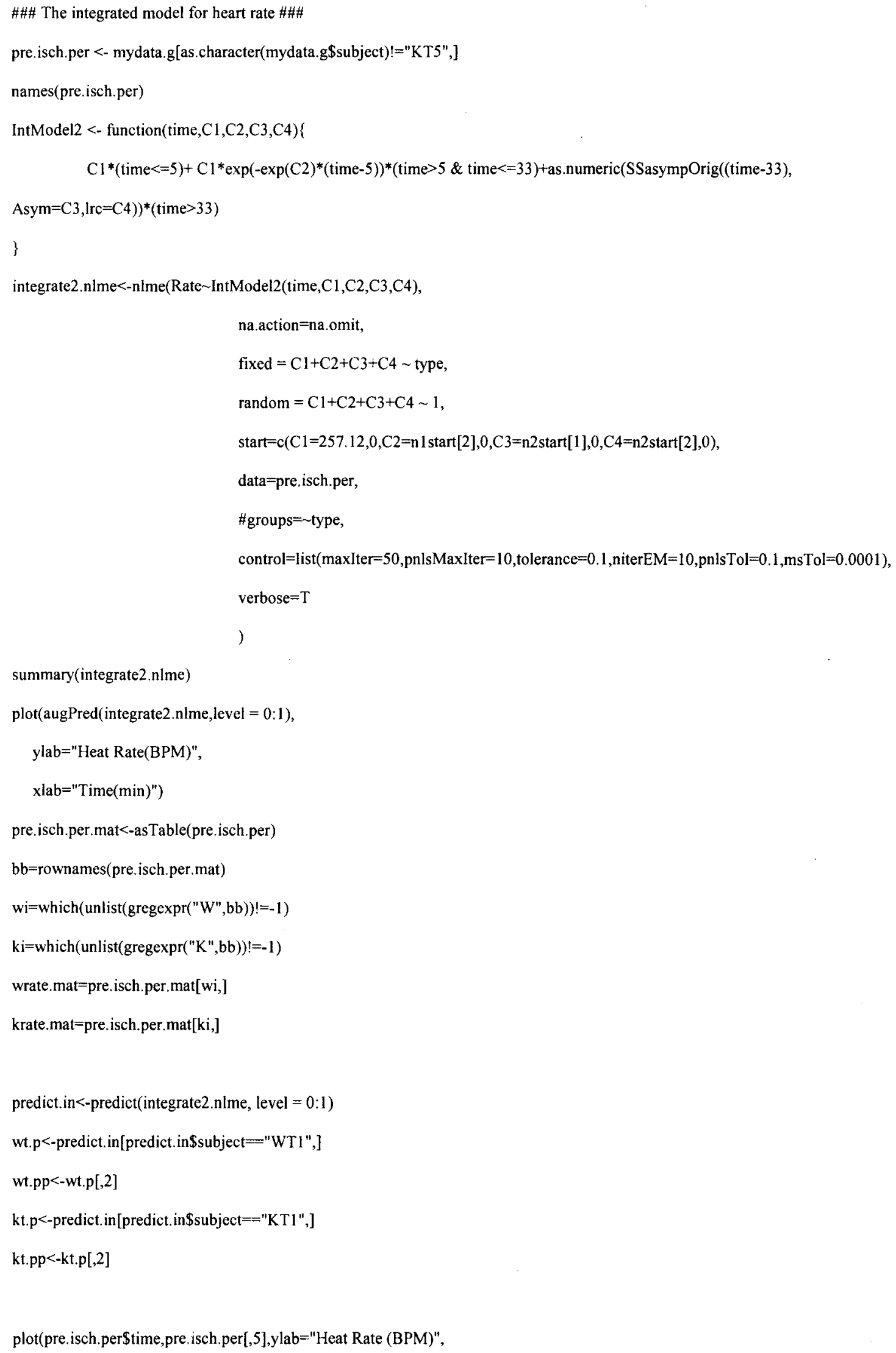




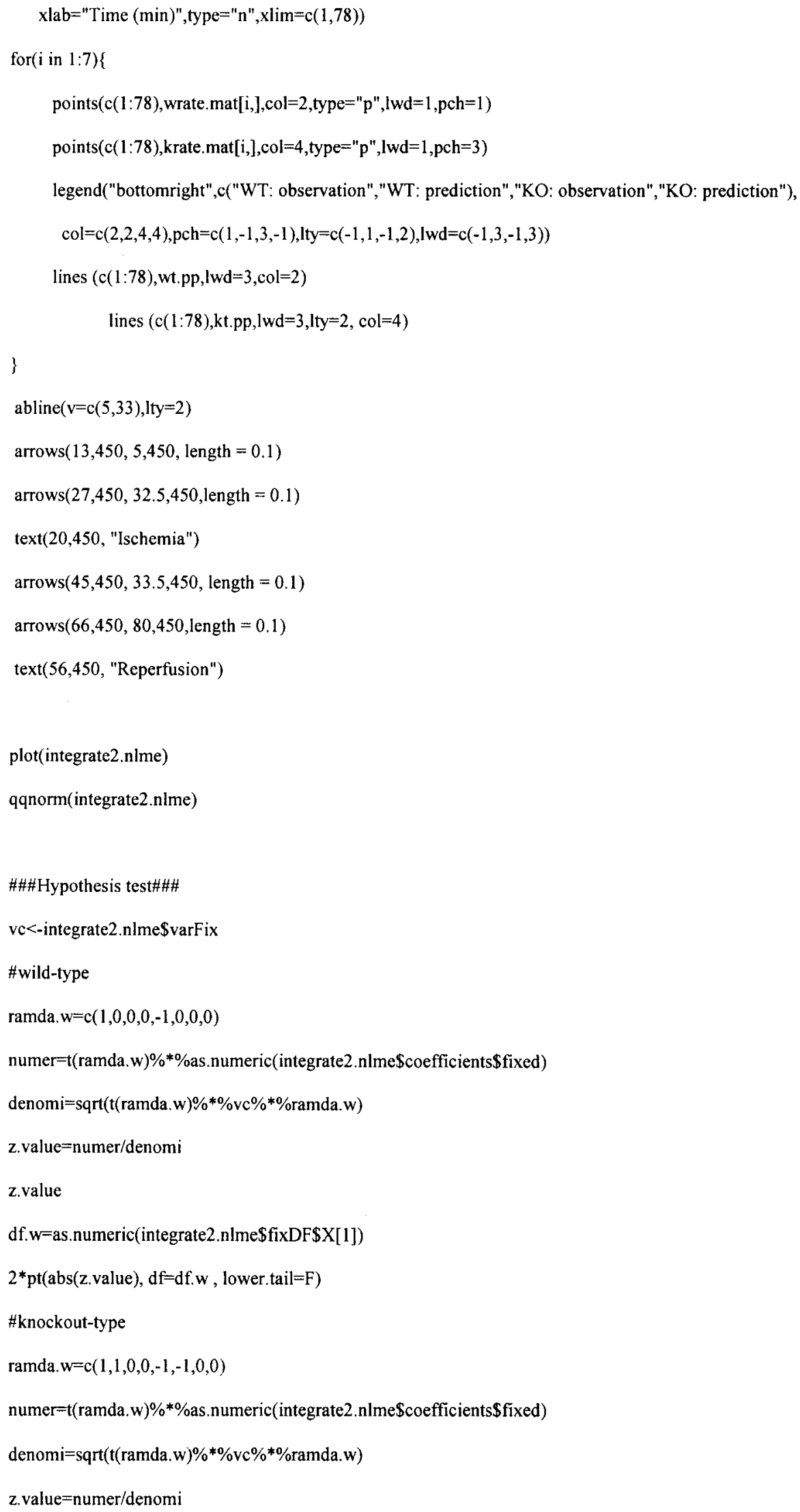




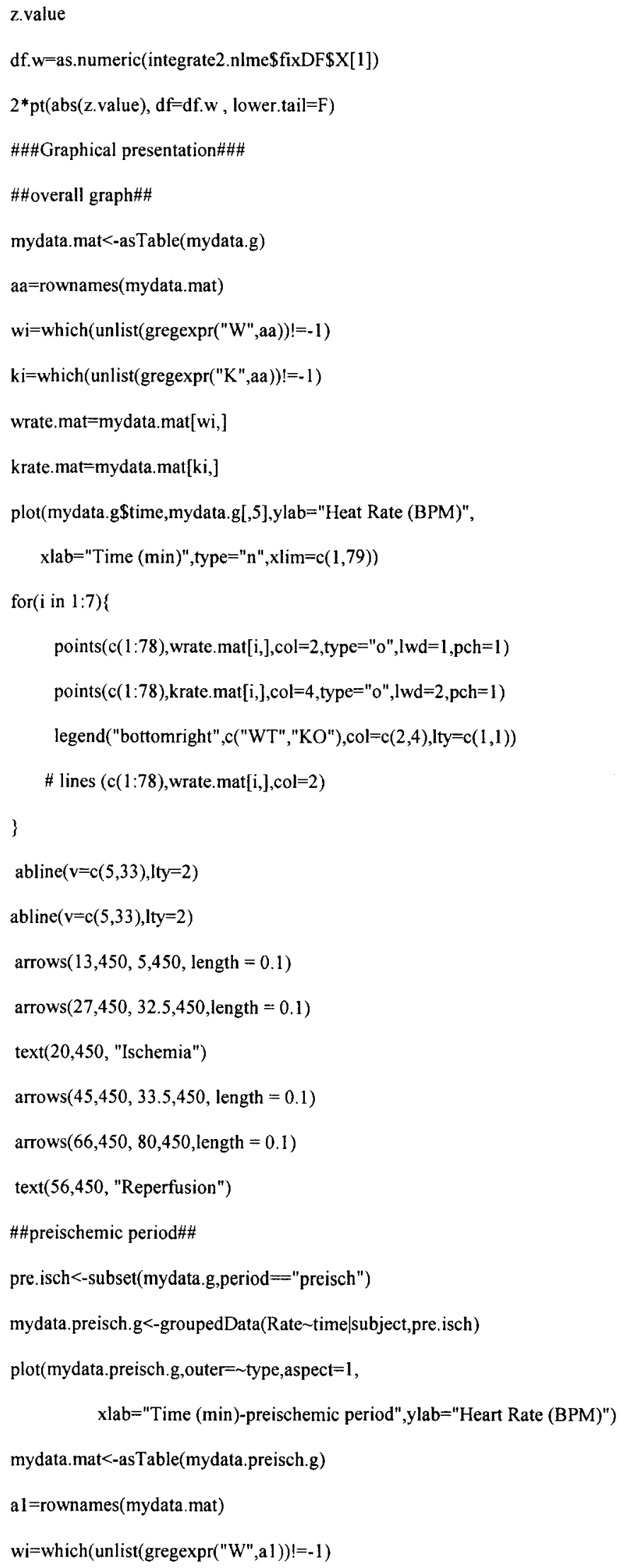




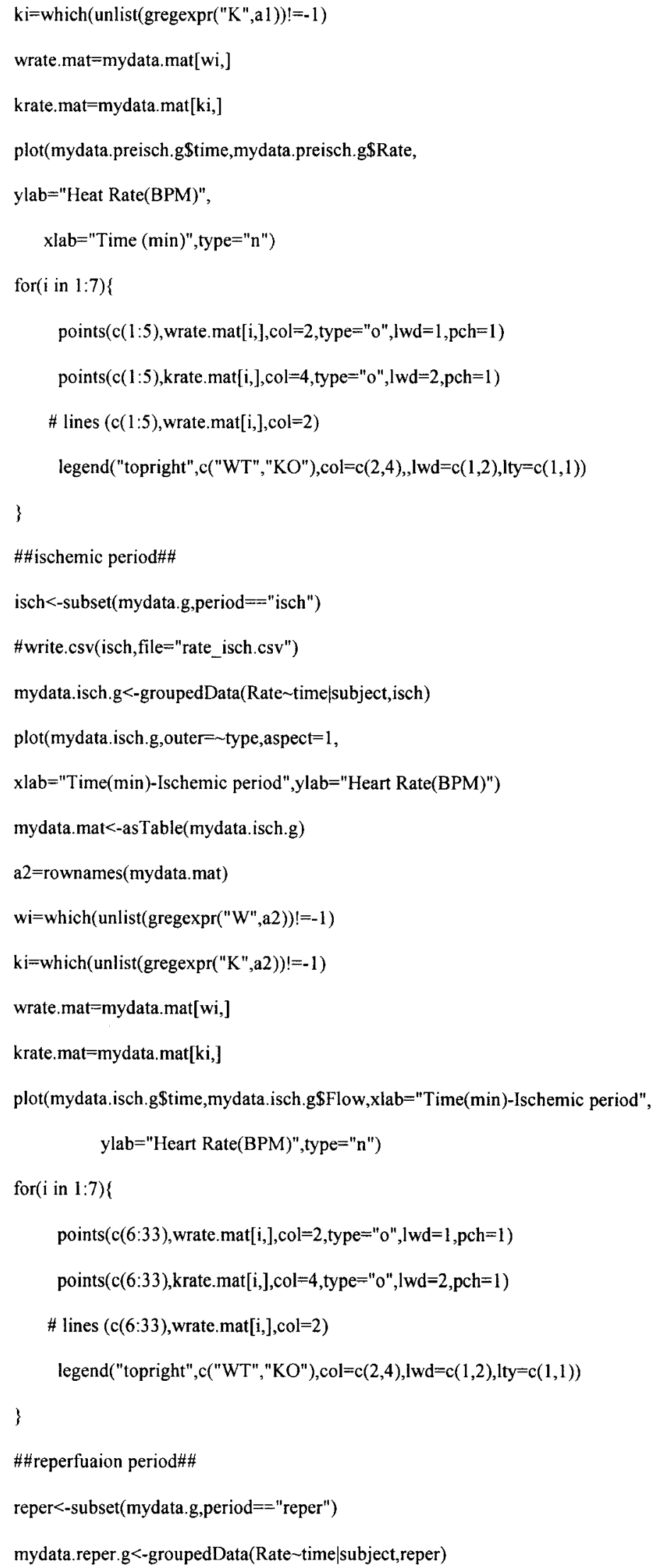




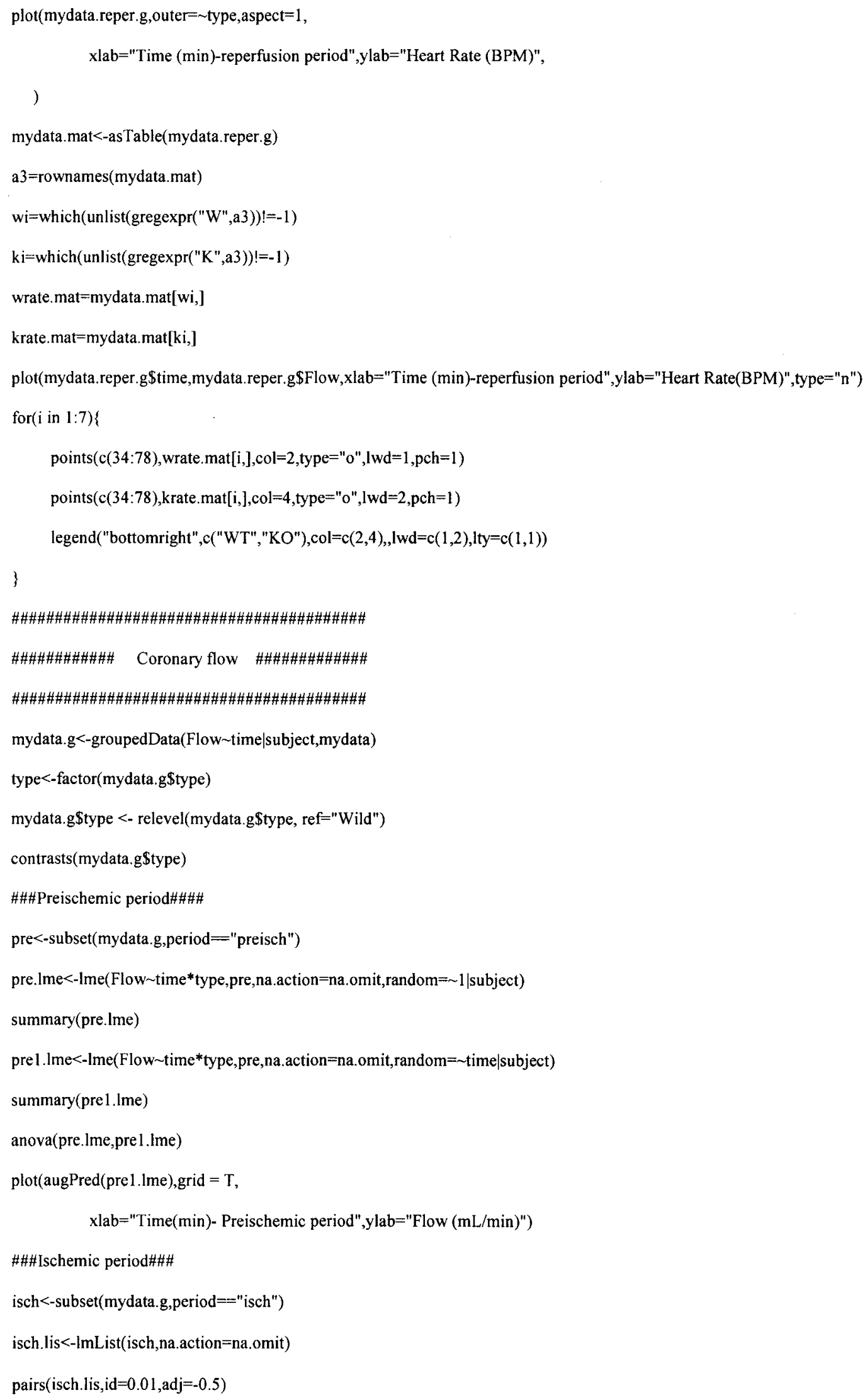




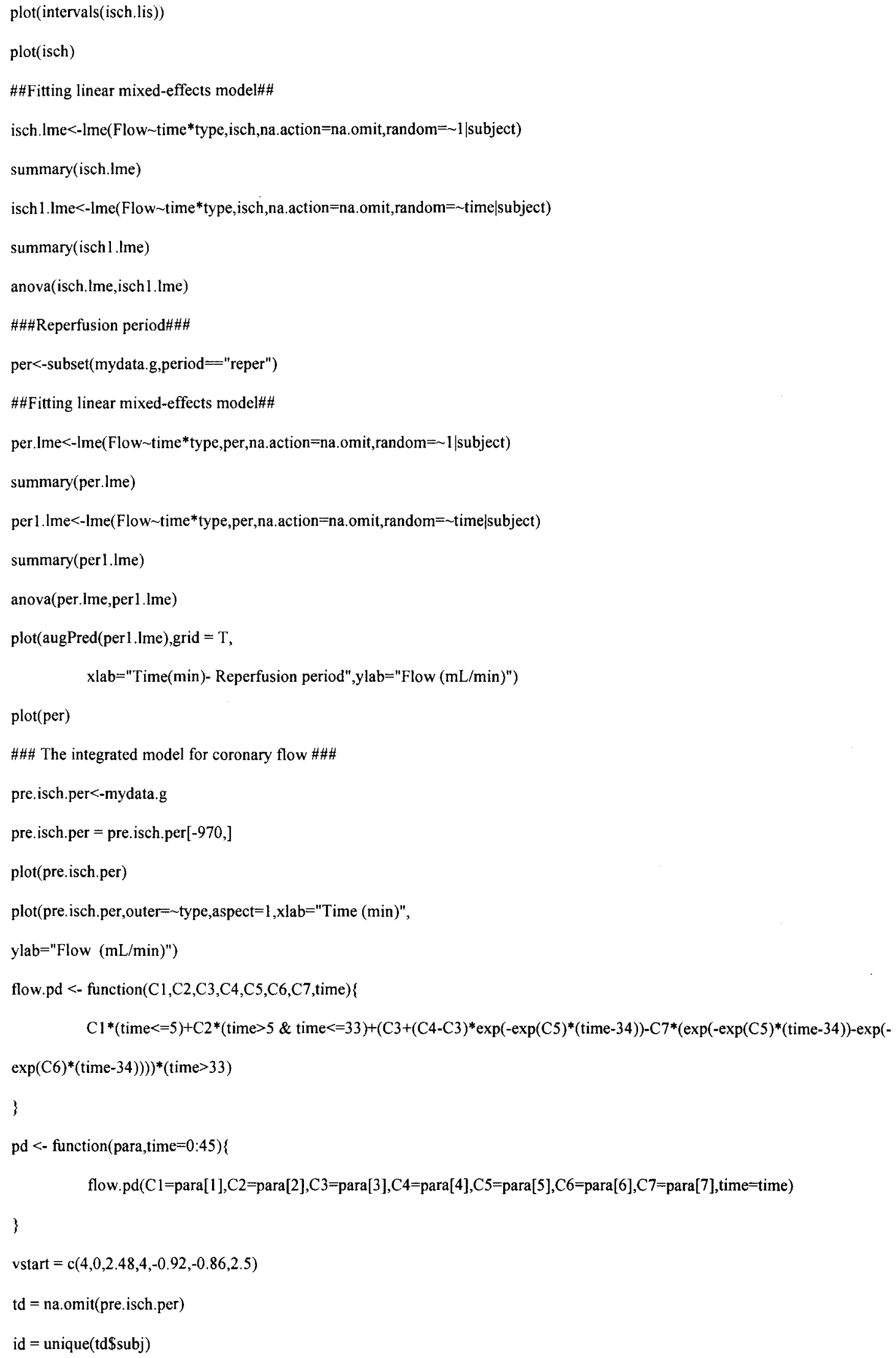




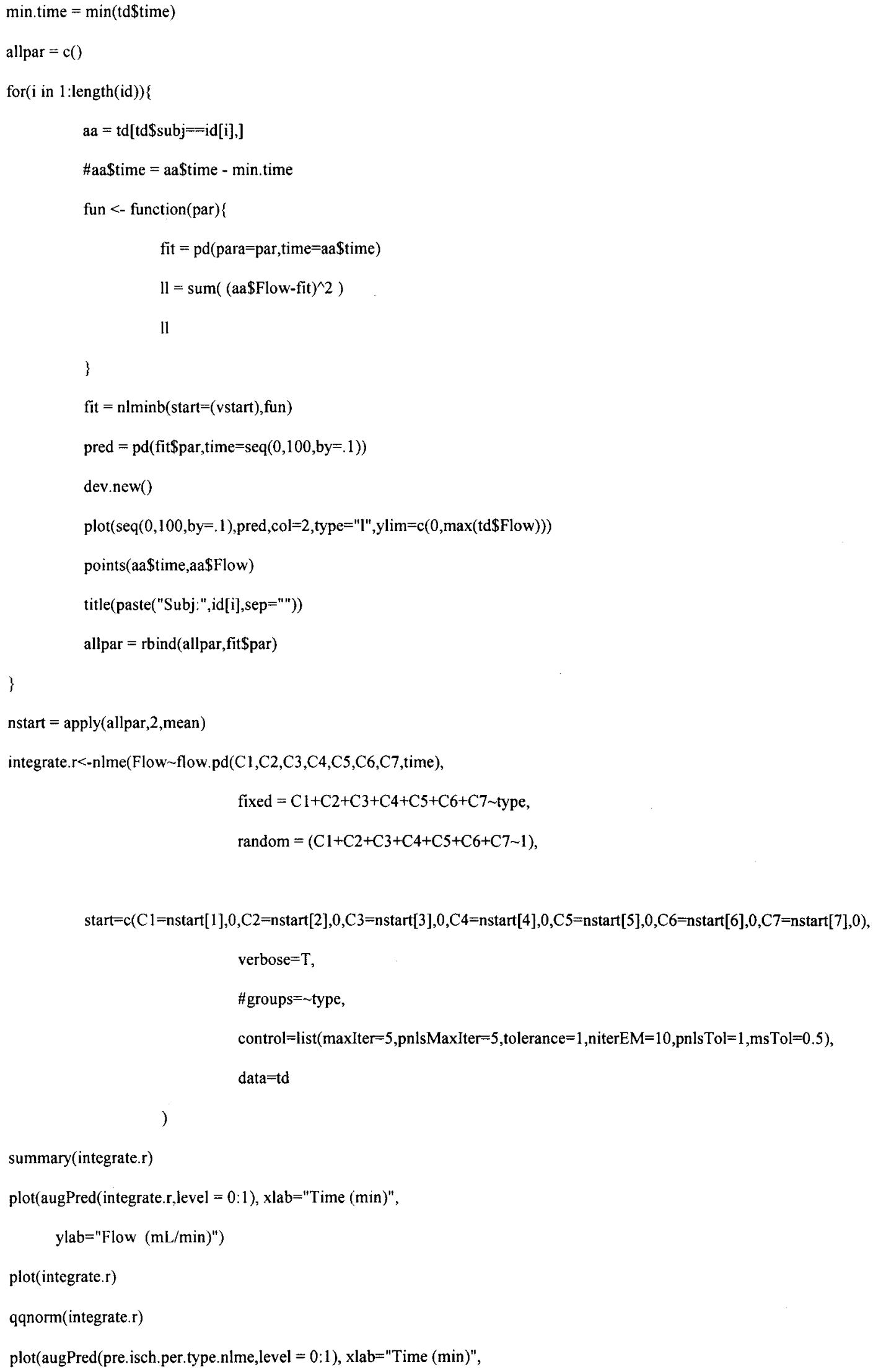


ylab="Flow $(\mathrm{mL} / \mathrm{min}) ")$

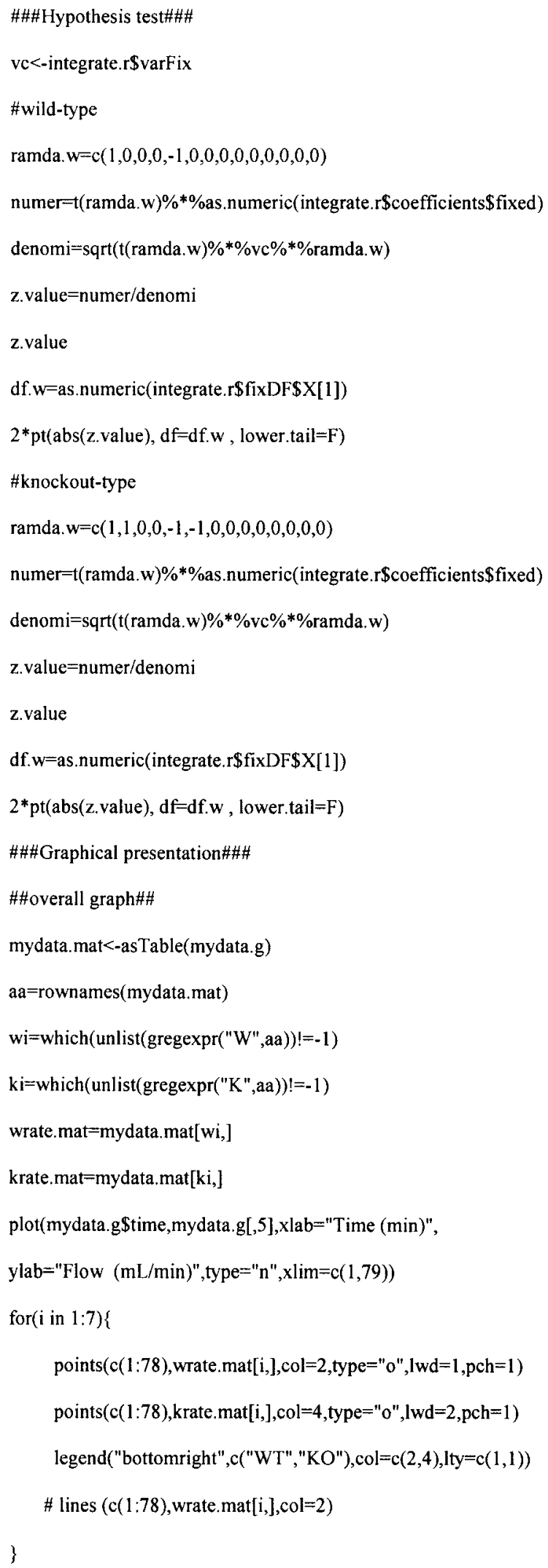




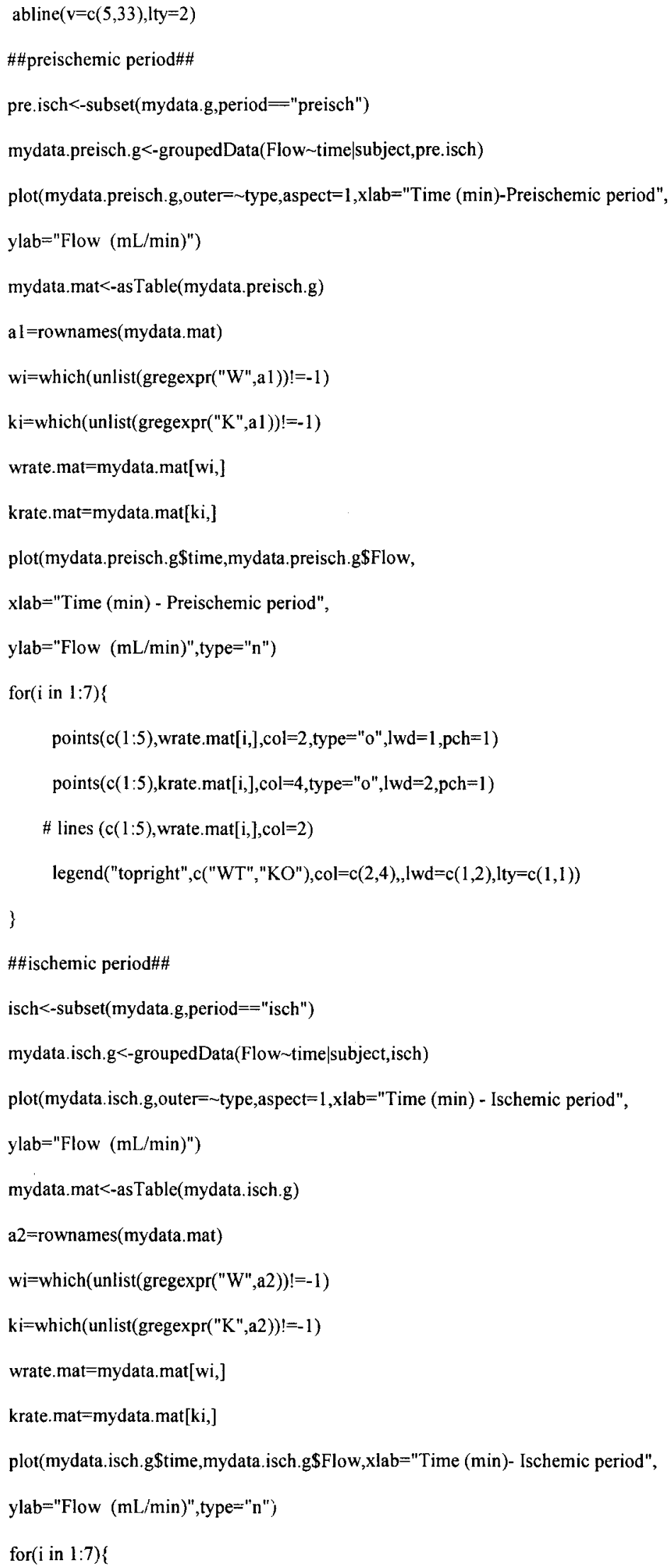




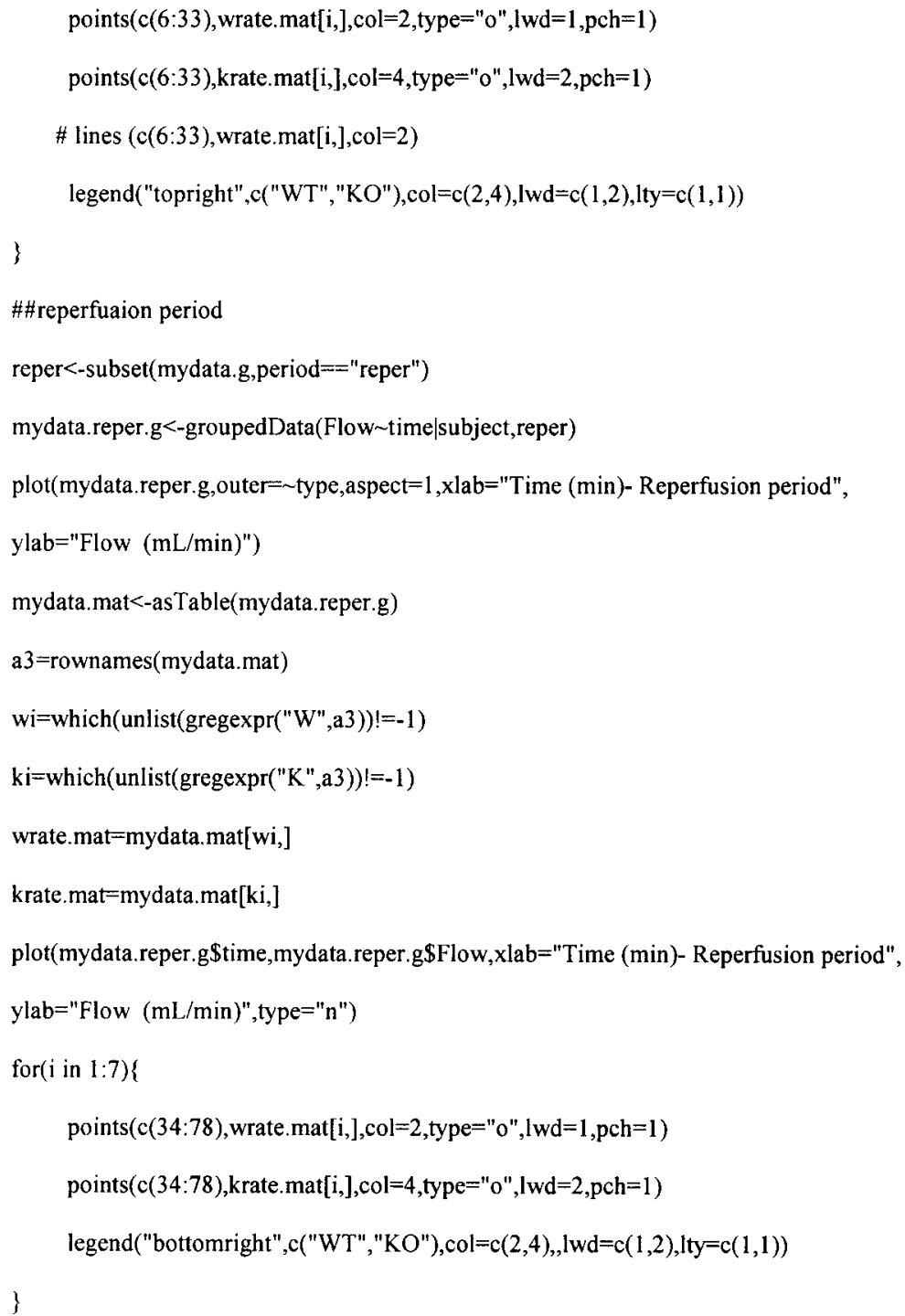




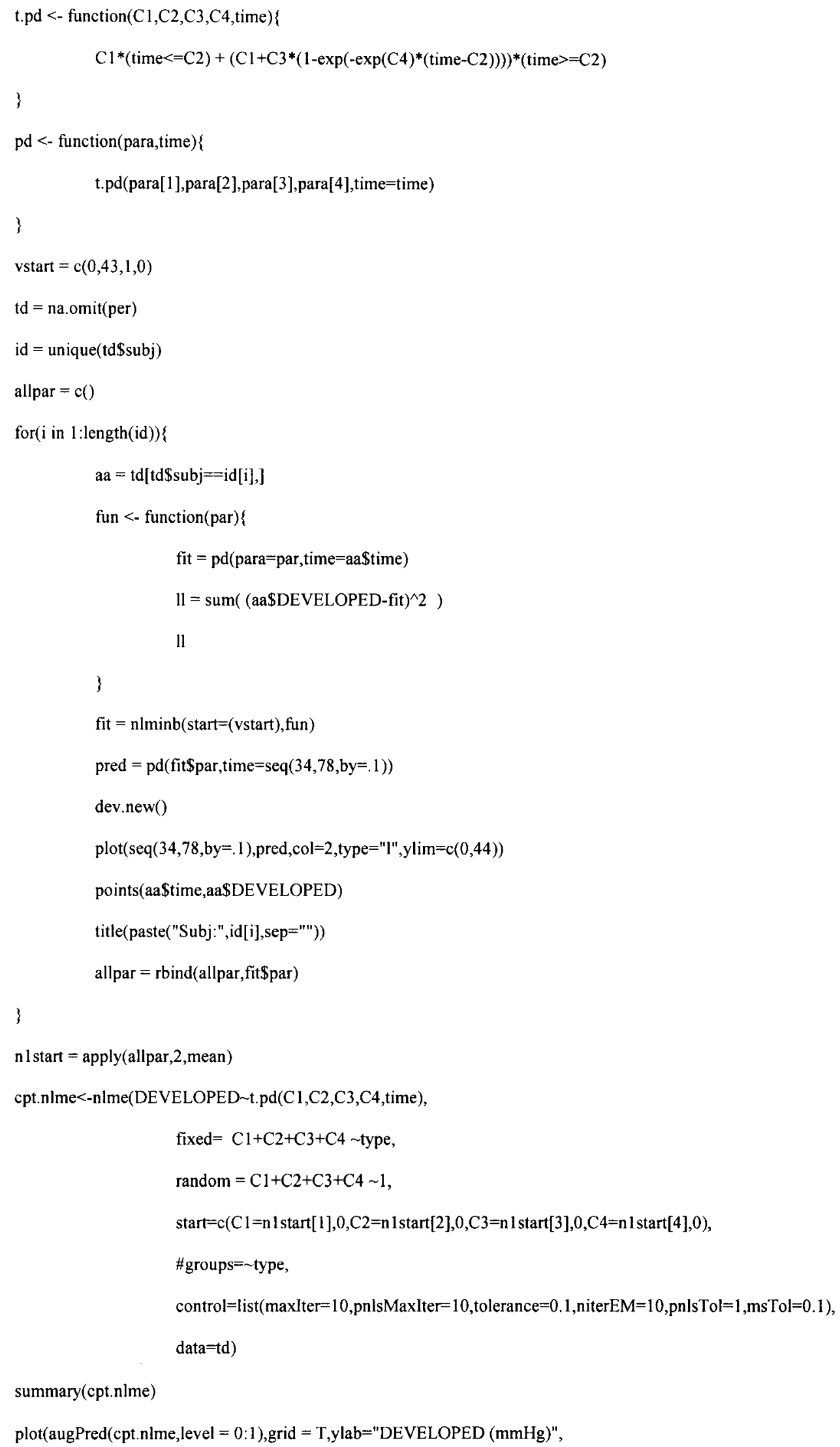


$\mathrm{xlab}=$ "Time(min)- Reperfusion period")

$\# \# \#$ Hypothesis test\#\#

vc<-cpt.nlme\$varFix $[4,4]$

beta.hat<-cpt.nlme\$coefficient\$fixed[4]

t.value $=$ beta.hat $/$ sqrt $(v c)$

\#wild-type

ramda. $w=c(0,0,0,1,0,0,0,0)$

numer=t(ramda.w)\%*\%as.numeric(integrate.r\$coefficients\$fixed)

denomi $=\operatorname{sqrt}($ t(ramda.w)\%*\%vc\%*\%ramda.w)

t.value $=$ numer $/ \mathrm{denomi}$

t.value

$\mathrm{df} . \mathrm{w}=$ as.numeric(integrate.r\$fixDF $\$[1])$

$2 * \operatorname{pt}($ abs(t.value), df=-df.w , lower.tail $=F)$

\#knockout-type

ramda. $w=c(0,0,0,0,0,1,0,0)$

numer-t(ramda.w)\%*\%as.numeric(integrate.r\$coefficients $\$$ fixed)

denomi $=$ sqrt(t(ramda. $w) \% * \% v c \% * \%$ ramda.w)

z.value $=$ numer $/$ denomi

z.value

df. $w=$ as.numeric (integrate. $\mathbf{r}$ fixDF $\$ \mathrm{X}[1])$

$2 * \operatorname{pt}($ abs(z.value), df=df.w , lower.tail=F)

\#\#\#Graphical presentation\#\#\#

\#\#overall graph\#\#

mydata.mat<-asTable(mydata.g)

aa=rownames(mydata.mat)

wi=which(unlist(gregexpr("W",aa))!=-1)

ki=which(unlist(gregexpr("K",aa))!=-1)

wrate. mat=mydata.mat[wi,]

krate.mat=mydata.mat[ki,]

plot(mydata.g\$time,mydata.g[,5],xlab="Time $(\min ) "$,

ylab="LV developed pressure $(\mathrm{mmHg})$ ",type="n",xlim=c(1,79))

for(i in 1:7)\{

points $(c(1: 78)$, wrate.mat[i,],col=2,type="o",lwd=1,pch=1)

points $(\mathrm{c}(1: 78), \mathrm{krate} \cdot \mathrm{mat}[\mathrm{i}], \mathrm{col}=4, \mathrm{type}=" \mathrm{o} ", \mathrm{lwd}=2, \mathrm{pch}=1)$

legend("topright",c("WT","KO"),col=c(2,4),lty=c(1,1)) 
\# lines $(\mathrm{c}(1: 78)$, wrate $\mathrm{mat}[\mathrm{i}],, \mathrm{col}=2)$ 


\section{CURRICULUM VITAE}

NAME: Hyejeong Jang

ADDRESS: Department of Bioinformatics and Biostatistics School of Public Health, University of Louisville Louisville, KY 40292

E-MAIL: webelieve75@gmail.com

EDUCATION:

B.S., Mathematics Education

March 1993 - Feb 1997

Chonnam National University, Gwangju, South Korea

CIRTIFICATES:

- National Teaching Certificate in Mathematics, 1998, Chonnam National University, 1998

- Certificate of Graduate Study in Mathematics Education, Chonnam National University, 2003

MEMBERSHIP:

- ASA membership since 2011

WORKING EXPERIENCE:

- Mathematics teacher, Unnam Middle school, Gwangju, South Korea, 2000- 2003

- Administration Assistant, Gwangju Metropolitan Office of Education, Gwangju, South Korea, 1999-2000

\section{RESEARCH EXPERIENCE:}

- Analysis of longitudinal data

Hyejeong Jang, Dr. Maying Kong. 
- ABSTRACT

Kong M, Jang $\mathrm{H}$, Conklin DJ. Mixed-effects models for evaluating cardiac function and treatment effect. ICSA Symposium June 26- 29, 2011, New York.

\section{COMPUTER SKILLS:}

- Familiar with SAS and R 